\title{
ALMOST DIAGONALIZATION OF $\tau$-PSEUDODIFFERENTIAL OPERATORS WITH SYMBOLS IN WIENER AMALGAM AND MODULATION SPACES
}

\author{
ELENA CORDERO, FABIO NICOLA, AND S. IVAN TRAPASSO
}

\begin{abstract}
In this paper we focus on the almost-diagonalization properties of $\tau$ pseudodifferential operators using techniques from time-frequency analysis. Our function spaces are modulation spaces and the special class of Wiener amalgam spaces arising by considering the action of the Fourier transform of modulation spaces. Such spaces are nowadays called modulation spaces as well. A particular example is provided by the Sjöstrand class, for which Gröchenig [Grö06] exhibits the almost diagonalization of Weyl operators. We shall show that such result can be extended to any $\tau$-pseudodifferential operator, for $\tau \in[0,1]$. This is not surprising, since the mapping that goes from a Weyl symbol to a $\tau$-symbol is bounded in the Sjöstrand class. What is new and quite striking is the almost diagonalization for $\tau$-operators with symbols in weighted Wiener amalgam spaces. In this case the diagonalization depends on the parameter $\tau$. In particular, we have an almost diagonalization for $\tau \in(0,1)$ whereas the cases $\tau=0$ or $\tau=1$ yield only to weaker results. As a consequence, we infer boundedness, algebra and Wiener properties for $\tau$-pseudodifferential operators on Wiener amalgam and modulation spaces.
\end{abstract}

\section{INTRODUCTION}

It is widely known that the techniques of Time-frequency Analysis revealed to be very fruitful when dealing with diverse problems in quite different fields, from signal processing to harmonic analysis, but also from PDE's to quantum mechanics (see, e.g., [BDD010, CN08, DdGP14, dG17, RWZ16, STW11, [STW11]). Besides the manifold achievements, Gabor analysis is a fascinating discipline in itself: the study of related functional-analytic problems is an inexhausted source of challenges and it happens to cast new light on established results, eventually. As an example, Gröchenig employed these methods in his work [Grö06], almost diagonalizing Weyl operators with symbols in the Sjöstrand class via Gabor frames. This result has provided new insights on a number of well-known outcomes previously obtained by

2010 Mathematics Subject Classification. 47G30,35S05,42B35,81S30.

Key words and phrases. Time-frequency analysis, $\tau$-Wigner distribution, $\tau$-pseudodifferential operators, almost diagonalization, modulation spaces, Wiener amalgam spaces. 
Sjöstrand within the realm of classical analysis [Sjö94, Sjö95] and, more importantly, has lead to far-reaching generalizations.

In this work we extend part of the results obtained in [Grö06] and derive a number of relevant consequences on the boundedness of pseudodifferential operators. In particular, our study has been carried out within the unifying framework offered by $\tau$-representations and $\tau$-pseudodifferential operators. $\tau$-pseudodifferential operators can be either defined as a quantization rule or by means of the related time-frequency representation (cf. [BDDO10, BCDDO10, BDDOC10]). In the following section we give a more detailed account, but here we simply recall the latter. For $\tau \in[0,1]$, the (cross-) $\tau$-Wigner distribution is given by

$$
W_{\tau}(f, g)(x, \omega)=\int_{\mathbb{R}^{d}} e^{-2 \pi i y \omega} f(x+\tau y) \overline{g(x-(1-\tau) y)} d y, \quad f, g \in \mathcal{S}\left(\mathbb{R}^{d}\right),
$$

whereas the $\tau$-pseudodifferential operator is defined by

$$
\left\langle\mathrm{Op}_{\tau}(a) f, g\right\rangle=\left\langle a, W_{\tau}(g, f)\right\rangle, \quad f, g \in \mathcal{S}\left(\mathbb{R}^{d}\right) .
$$

For $\tau=1 / 2$ we recapture the Weyl operator studied in Grö06, if $\tau=0$ the operator is called the Kohn-Nirenberg operator $\mathrm{Op}_{\mathrm{KN}}$, if $\tau=1$ the related operator is named operator "with right symbol".

For what concerns the symbol classes, we consider Banach spaces allowing a suitable measure of the time-frequency decay of distributions, i.e. modulation and Wiener amalgam spaces. These function spaces were introduced by Feichtinger in the '80s (cf. [Fei81, Fei83]) and revealed to be the optimal setting for a large class of problems related to harmonic analysis and PDE's (cf. dG11, dGGR16, WHHG11]).

To fix notation, we write a point in phase space (in time-frequency space) as $z=(x, \omega) \in \mathbb{R}^{2 d}$, and the corresponding phase-space shift (time-frequency shift) acting on a function or distribution as

$$
\pi(z) f(t)=e^{2 \pi i \omega t} f(t-x), \quad t \in \mathbb{R}^{d} .
$$

When considering symbol functions, we shall work with time-frequency shifts $\pi(z, \zeta)$, with $z, \zeta \in \mathbb{R}^{2 d}$ (the variables are doubled).

Consider a Schwartz function $g \in \mathcal{S}\left(\mathbb{R}^{2 d}\right) \backslash\{0\}$. We define the modulation space $M^{\infty, 1}\left(\mathbb{R}^{2 d}\right)$ (or Sjöstrand class) as the space of tempered distributions $\sigma \in \mathcal{S}^{\prime}\left(\mathbb{R}^{2 d}\right)$ such that

$$
\int_{\mathbb{R}^{2 d}} \sup _{z \in \mathbb{R}^{2 d}}|\langle\sigma, \pi(z, \zeta) g\rangle| d \zeta<\infty .
$$

For the properties of such space and its weighted versions we refer to the next section. Here the main focus is on symbols in the so-called Wiener amalgam space $W\left(\mathcal{F} L^{\infty}, L^{1}\right)\left(\mathbb{R}^{2 d}\right)=\mathcal{F} M^{\infty, 1}\left(\mathbb{R}^{2 d}\right)$, where $\mathcal{F}$ is the Fourier transform. Heuristically, symbols in $W\left(\mathcal{F} L^{\infty}, L^{1}\right)\left(\mathbb{R}^{2 d}\right)$ display locally a regularity of the type $\mathcal{F} L^{\infty}\left(\mathbb{R}^{2 d}\right)$ and globally decay as functions in $L^{1}\left(\mathbb{R}^{2 d}\right)$. For instance, the $\delta$ distribution $\left(\right.$ in $\mathcal{S}^{\prime}\left(\mathbb{R}^{2 d}\right)$ ) 
belongs to $W\left(\mathcal{F} L^{\infty}, L^{1}\right)\left(\mathbb{R}^{2 d}\right)$. Nowadays this class can be referred to as modulation space as well (although in a generalized sense, as suggested by Feichtinger in his retrospective [Fei06]), see also [MP13.

The central issue of our work is the approximate diagonalization of $\tau$-pseudodifferential operators. This is a well-known problem, studied in several contexts of harmonic analysis: classical references are [Mey90, RT98]. See also, for the more general framework of Fourier integral operators, [GL08, CGNR13] and references therein. In somewhat heuristic terms, the choice of certain type of symbols assures that these operators preserve the time-frequency localization, since their kernel with respect to continuous or discrete time-frequency shifts satisfies a convenient decay condition. To be precise, thanks to a covariance property for the $\tau$-Wigner distribution under the action of time-frequency shifts, we are able to prove the following claim, which extends [Grö06, Thereom 3.2] proved for $\tau=\frac{1}{2}$, i.e., for the Weyl quantization (see the subsequent Theorem 4.1 for the weighted version).

First, we recall the definition of a Gabor frame. Let $\Lambda=A \mathbb{Z}^{2 d}$, with $A \in$ $\mathrm{GL}(2 d, \mathbb{R})$, be a lattice of the time-frequency plane. The set of time-frequency shifts $\mathcal{G}(\varphi, \Lambda)=\{\pi(\lambda) \varphi: \lambda \in \Lambda\}$ for a non-zero $\varphi \in L^{2}\left(\mathbb{R}^{d}\right)$ (the so-called window function) is named a Gabor system. The set $\mathcal{G}(\varphi, \Lambda)$ is a Gabor frame, if there exist constants $A, B>0$ such that

$$
A\|f\|_{2}^{2} \leq \sum_{\lambda \in \Lambda}|\langle f, \pi(\lambda) \varphi\rangle|^{2} \leq B\|f\|_{2}^{2}, \quad \forall f \in L^{2}\left(\mathbb{R}^{d}\right) .
$$

Our result is the following:

Theorem. Fix a non-zero window $\varphi \in \mathcal{S}\left(\mathbb{R}^{d}\right)$ such that $\mathcal{G}(\varphi, \Lambda)$ is a Gabor frame for $L^{2}\left(\mathbb{R}^{d}\right)$. For any $\tau \in[0,1]$, the following properties are equivalent:

(i) $\sigma \in M^{\infty, 1}\left(\mathbb{R}^{2 d}\right)$.

(ii) $\sigma \in \mathcal{S}^{\prime}\left(\mathbb{R}^{2 d}\right)$ and there exists a function $H_{\tau} \in L^{1}\left(\mathbb{R}^{2 d}\right)$ such that

$$
\left|\left\langle\mathrm{Op}_{\tau}(\sigma) \pi(z) \varphi, \pi(w) \varphi\right\rangle\right| \leq H_{\tau}(w-z), \quad \forall w, z \in \mathbb{R}^{2 d} .
$$

(iii) $\sigma \in \mathcal{S}^{\prime}\left(\mathbb{R}^{2 d}\right)$ and there exists a sequence $h_{\tau} \in \ell^{1}(\Lambda)$ such that

$$
\left|\left\langle\mathrm{Op}_{\tau}(\sigma) \pi(\mu) \varphi, \pi(\lambda) \varphi\right\rangle\right| \leq h_{\tau}(\lambda-\mu), \quad \forall \lambda, \mu \in \Lambda .
$$

This kind of control is a characterizing property of symbols in those spaces, in the following sense.

Corollary. Under the hypotheses of the previous Theorem, let $\tau \in[0,1]$ be fixed and assume that $T: \mathcal{S}\left(\mathbb{R}^{d}\right) \rightarrow \mathcal{S}^{\prime}\left(\mathbb{R}^{d}\right)$ is continuous and satisfies one of the following conditions:

(i) $|\langle T \pi(z) \varphi, \pi(w) \varphi\rangle| \leq H(w-z), \quad \forall w, z \in \mathbb{R}^{2 d}$ for some $H \in L^{1}$.

(ii) $|\langle T \pi(\mu) \varphi, \pi(\lambda) \varphi\rangle| \leq h(\lambda-\mu), \quad \forall \lambda, \mu \in \Lambda$ for some $h \in \ell^{1}$.

Therefore, $T=\mathrm{Op}_{\tau}(\sigma)$ for some symbol $\sigma \in M^{\infty, 1}\left(\mathbb{R}^{2 d}\right)$. 
We remark that, if either the previous condition $(i)$ or $(i i)$ is satisfied, then it is well known that the operator $T$ has Weyl symbol in the Sjöstrand's class ([Grö06, Thereom 3.2]). On the other hand, the mapping that goes to a Weyl symbol to a $\tau$ symbol is bounded in the Sjöstrand's class, and the previous corollary follows from Grö06, Thereom 3.2]. Novelty in this connection mainly resides in the weighted version, cf. Theorem 4.1 and Corollary 4.2 below.

A parallel pattern can be traced for symbols in $W\left(\mathcal{F} L^{\infty}, L^{1}\right)\left(\mathbb{R}^{2 d}\right)$ although it is somewhat more involved because of the peculiar way $\tau$ comes across. This is not surprising if we notice that Weyl operators with symbols in $L^{1}\left(\mathbb{R}^{2 d}\right) \subset$ $W\left(\mathcal{F} L^{\infty}, L^{1}\right)\left(\mathbb{R}^{2 d}\right)$ are bounded on $L^{2}\left(\mathbb{R}^{d}\right)$ (cf. WHHG11) but neither KohnNirenberg operators $(\tau=0)$ nor operators "with right $\operatorname{symbol}^{\prime}(\tau=1)$ are, cf. [Bou95, Bou97a, Bou97b], see also [CDNT18].

For $\tau \in(0,1)$, we introduce the symplectic matrix

$$
\mathcal{U}_{\tau}=-\left(\begin{array}{cc}
\frac{\tau}{1-\tau} I_{d \times d} & 0_{d \times d} \\
0_{d \times d} & \frac{1-\tau}{\tau} I_{d \times d}
\end{array}\right) \in \operatorname{Sp}(2 d, \mathbb{R})
$$

(see the next section for details). Our result is as follows:

Theorem. Fix non-zero window $\varphi \in \mathcal{S}\left(\mathbb{R}^{d}\right)$. For any $\tau \in(0,1)$, the following properties are equivalent:

(i) $\sigma \in W\left(\mathcal{F} L^{\infty}, L^{1}\right)\left(\mathbb{R}^{2 d}\right)$.

(ii) $\sigma \in \mathcal{S}^{\prime}\left(\mathbb{R}^{2 d}\right)$ and there exists a function $H_{\tau} \in L^{1}\left(\mathbb{R}^{2 d}\right)$ such that

$$
\left|\left\langle\mathrm{Op}_{\tau}(\sigma) \pi(z) \varphi, \pi(w) \varphi\right\rangle\right| \leq H_{\tau}\left(w-\mathcal{U}_{\tau} z\right), \quad \forall w, z \in \mathbb{R}^{2 d} .
$$

As easy example, we shall consider $\sigma=\delta \in W\left(\mathcal{F} L^{\infty}, L^{1}\right)$ (cf. Example 4.4 below). In this case, it is easy to see that $\mathrm{Op}_{\tau}(\delta)$ is a linear change of variables and the estimate in (6) is an equality.

Another difference from the Sjöstrand symbol class concerns the discrete almost diagonalization, recovered only if $\tau=\frac{1}{2}$, see the subsequent Corollary 4.5.

Even though

$$
w-\mathcal{U}_{\tau} z=0
$$

is not exactly the diagonal, by abuse of notation we use the term almost diagonalization also in this framework. In fact, (6) can be suitably interpreted as an estimate on the concentration of the time-frequency representation $\mathrm{Op}_{\tau}(\sigma)$ along the graph of $\mathcal{U}_{\tau}$.

This kind of control on the kernels paves the way for proving relevant properties of $\tau$-operators. Here we mainly focus on boundedness results in different settings, where Wiener amalgam and modulation spaces play the role of symbol class and domain/target. In particular, we underline the connection with the theory of Fourier integral operators (FIOs): we have that, under suitable conditions, $\tau$-pseudodifferential operators admit a representation as type I FIOs, thus 
inheriting a number of features (including boundedness, algebra and Wiener properties) established in previous papers by two of the authors - see for instance CNR09, CGNR13, CGNR14. In addition, we point out that even when this overlapping does not hold, the insightful proof strategies developed there can still be exploited. Apart from the technical assumptions on weights which will be specified later, our results can be collected in Table 1 .

\begin{tabular}{|c|c|c|}
\hline $\boldsymbol{\tau}$ & Symbol - $\left(\mathbb{R}^{2 d}\right)$ & Boundedness - $\left(\mathbb{R}^{d}\right), 1 \leq p, q \leq \infty$ \\
\hline \hline$(0,1)$ & $\sigma \in W\left(\mathcal{F} L^{\infty}, L^{1}\right)$ & $\mathrm{Op}_{\tau}(\sigma): M^{p, q} \rightarrow M^{p, q}$ \\
\hline$[0,1]$ & $\sigma \in M^{\infty, 1}$ & $\mathrm{Op}_{\tau}(\sigma): W\left(\mathcal{F} L^{p}, L^{q}\right) \rightarrow W\left(\mathcal{F} L^{p}, L^{q}\right)$ \\
\hline$(0,1)$ & $\sigma \in W\left(\mathcal{F} L^{\infty}, L^{1}\right)$ & $\mathrm{Op}_{\tau}(\sigma): W\left(\mathcal{F} L^{p}, L^{q}\right) \rightarrow W\left(\mathcal{F} L^{p}, L^{q}\right)$ \\
\hline 0 & $\sigma \in W\left(\mathcal{F} L^{\infty}, L^{1}\right)$ & $\mathrm{Op}_{\mathrm{KN}}(\sigma): M^{1, \infty} \rightarrow M^{1, \infty}$ \\
\hline 1 & $\sigma \in W\left(\mathcal{F} L^{\infty}, L^{1}\right)$ & $\mathrm{Op}_{1}(\sigma): W\left(\mathcal{F} L^{1}, L^{\infty}\right) \rightarrow W\left(\mathcal{F} L^{1}, L^{\infty}\right)$ \\
\hline
\end{tabular}

TABLE 1. Boundedness results proved in the paper.

It is important to emphasize again the singular behaviour showed by the endpoints $\tau=0$ and $\tau=1$, which is essentially due to weaker versions of the previous theorems, see Propositions 5.8 and 5.9 in the sequel.

We finally remark that, as said earlier, the results on approximate diagonalization allow a potentially large number of directions to be investigated, even in a more abstract framework (cf. for instance [GR08]). We wish to explore some of these routes in subsequent papers.

\section{Preliminaries}

Notation. We define $t^{2}=t \cdot t$, for $t \in \mathbb{R}^{d}$, and $x y=x \cdot y$ is the scalar product on $\mathbb{R}^{d}$. The Schwartz class is denoted by $\mathcal{S}\left(\mathbb{R}^{d}\right)$, the space of tempered distributions by $\mathcal{S}^{\prime}\left(\mathbb{R}^{d}\right)$. We use the brackets $\langle f, g\rangle$ to denote the extension to $\mathcal{S}^{\prime}\left(\mathbb{R}^{d}\right) \times \mathcal{S}\left(\mathbb{R}^{d}\right)$ of the inner product $\langle f, g\rangle=\int f(t) \overline{g(t)} d t$ on $L^{2}\left(\mathbb{R}^{d}\right)$. The Fourier transform of a function $f$ on $\mathbb{R}^{d}$ is normalized as

$$
\mathcal{F} f(\xi)=\int_{\mathbb{R}^{d}} e^{-2 \pi i x \xi} f(x) d x .
$$

The modulation $M_{\omega}$ and translation $T_{x}$ operators are defined as

$$
M_{\omega} f(t)=e^{2 \pi i t \omega} f(t), \quad T_{x} f(t)=f(t-x) .
$$


The following result exhibits composition and commutation properties of the timefrequency shifts.

Lemma 2.1. For $x, x^{\prime}, \omega, \omega^{\prime} \in \mathbb{R}^{d}$, we have

(i) Commutation relations for TF-shifts:

$$
T_{x} M_{\omega}=e^{-2 \pi i x \omega} M_{\omega} T_{x} .
$$

(ii) Compositions of TF-shifts:

$$
\pi(x, \omega) \pi\left(x^{\prime}, \omega^{\prime}\right)=e^{-2 \pi i x \omega^{\prime}} \pi\left(x+x^{\prime}, \omega+\omega^{\prime}\right) .
$$

More explicitly,

$$
\left(M_{\omega} T_{x}\right)\left(M_{\omega^{\prime}} T_{x^{\prime}}\right)=e^{-2 \pi i x \omega^{\prime}} M_{\omega+\omega^{\prime}} T_{x+x^{\prime}} .
$$

Let $f \in \mathcal{S}^{\prime}\left(\mathbb{R}^{d}\right)$. We define the short-time Fourier transform of $f$ as

$$
V_{g} f(x, \omega)=\langle f, \pi(x, \omega) g\rangle=\mathcal{F}\left(f T_{x} g\right)(\omega)=\int_{\mathbb{R}^{d}} f(y) \overline{g(y-x)} e^{-2 \pi i y \omega} d y .
$$

Recall the fundamental property of time-frequency analysis

$$
V_{g} f(x, \omega)=e^{-2 \pi i x \omega} V_{\hat{g}} \hat{f}(\omega,-x) .
$$

Denote by $J$ the canonical symplectic matrix in $\mathbb{R}^{2 d}$.

$$
J=\left(\begin{array}{cc}
0_{d \times d} & I_{d \times d} \\
-I_{d \times d} & 0_{d \times d}
\end{array}\right) \in \operatorname{Sp}(2 d, \mathbb{R}),
$$

where the symplectic group $\operatorname{Sp}(2 d, \mathbb{R})$ is defined by

$$
\operatorname{Sp}(2 d, \mathbb{R})=\left\{M \in \mathrm{GL}(2 d, \mathbb{R}): M^{\top} J M=J\right\} .
$$

Observe that, for $z=\left(z_{1}, z_{2}\right) \in \mathbb{R}^{2 d}$, we have $J z=J\left(z_{1}, z_{2}\right)=\left(z_{2},-z_{1}\right), J^{-1} z=$ $J^{-1}\left(z_{1}, z_{2}\right)=\left(-z_{2}, z_{1}\right)=-J z$, and $J^{2}=-I_{2 d \times 2 d}$.

Lemma 2.2. For any $\tau \in(0,1)$, consider the matrix

$$
\mathcal{A}_{\tau}=\left(\begin{array}{cc}
0_{d \times d} & \sqrt{\frac{1-\tau}{\tau}} I_{d \times d} \\
-\sqrt{\frac{\tau}{1-\tau}} I_{d \times d} & 0_{d \times d}
\end{array}\right) .
$$

The following properties hold:

(1) $\mathcal{A}_{\tau} \in \operatorname{Sp}(d, \mathbb{R})$; in particular, $\mathcal{A}_{1 / 2}=J$.

(2) $\mathcal{A}_{\tau}^{\top}=-\mathcal{A}_{1-\tau}, \mathcal{A}_{\tau}^{-1}=-\mathcal{A}_{\tau}$.

(3) $\mathcal{A}_{1-\tau} \mathcal{A}_{\tau}=\mathcal{A}_{\tau}^{\top} \mathcal{A}_{\tau}^{-1}=I_{2 d \times 2 d}-\mathcal{B}_{\tau}$, where

$$
\mathcal{B}_{\tau}=\left(\begin{array}{cc}
\frac{1}{1-\tau} I_{d \times d} & 0_{d \times d} \\
0_{d \times d} & \frac{1}{\tau} I_{d \times d}
\end{array}\right)
$$

(4) $\sqrt{\tau(1-\tau)}\left(\mathcal{A}_{\tau}+\mathcal{A}_{1-\tau}\right)=\sqrt{\tau(1-\tau)} \mathcal{B}_{\tau} \mathcal{A}_{\tau}=J$.

Proof. These properties follow by easy computations. 
Although $\mathcal{A}_{\tau}$ is well defined only for $\tau \in(0,1)$, notice that the matrix

$$
\mathcal{A}_{\tau}^{\prime}=\left(\begin{array}{cc}
0_{d \times d} & (1-\tau) I_{d \times d} \\
-\tau I_{d \times d} & 0_{d \times d}
\end{array}\right)
$$

is well defined for any $\tau \in[0,1]$, though non-invertible and non-symplectic when $\tau \in\{0,1\}$. In particular:

(1) For $\tau \in(0,1), \mathcal{A}_{\tau}^{\prime}=\sqrt{\tau(1-\tau)} \mathcal{A}_{\tau}$.

(2) $\left(\mathcal{A}_{\tau}^{\prime}\right)^{\top}=-\mathcal{A}_{1-\tau}^{\prime}$.

(3) $\mathcal{A}_{\tau}^{\prime}+\mathcal{A}_{1-\tau}^{\prime}=J$.

In the following we shall use the short-time Fourier transform of a $\tau$-Wigner distribution, which is contained in CDNT18.

Lemma 2.3. Consider $\tau \in[0,1], \varphi_{1}, \varphi_{2} \in \mathcal{S}\left(\mathbb{R}^{d}\right), f, g \in \mathcal{S}\left(\mathbb{R}^{d}\right)$ and set $\Phi_{\tau}=$ $W_{\tau}\left(\varphi_{1}, \varphi_{2}\right) \in \mathcal{S}\left(\mathbb{R}^{2 d}\right)$. Then

$$
V_{\Phi_{\tau}} W_{\tau}(g, f)(z, \zeta)=e^{-2 \pi i z_{2} \zeta_{2}} V_{\varphi_{1}} g\left(z-\mathcal{A}_{1-\tau}^{\prime} \zeta\right) \overline{V_{\varphi_{2}} f\left(z+\mathcal{A}_{\tau}^{\prime} \zeta\right)}
$$

where $z=\left(z_{1}, z_{2}\right), \zeta=\left(\zeta_{1}, \zeta_{2}\right) \in \mathbb{R}^{2 d}$ and the matrix $\mathcal{A}_{\tau}^{\prime}$ is defined in (13). In particular,

$$
\left|V_{\Phi_{\tau}} W_{\tau}(g, f)\right|=\left|V_{\varphi_{1}} g\left(z-\mathcal{A}_{1-\tau}^{\prime} \zeta\right)\right| \cdot\left|V_{\varphi_{2}} f\left(z+\mathcal{A}_{\tau}^{\prime} \zeta\right)\right|
$$

2.1. Function Spaces. We first need to introduce the weight functions under our consideration.

Weight functions. Following [Grö06, Sec. 2.3], we will work with so-called admissible weight functions, i.e. by $v$ we always denote a non-negative continuous function on $\mathbb{R}^{2 d}$ such that

(1) $v(0)=1$ and $v$ is even in each coordinate:

$$
v\left( \pm z_{1}, \ldots, \pm z_{2 d}\right)=v\left(z_{1}, \ldots, z_{2 d}\right) .
$$

(2) $v$ is submultiplicative, that is

$$
v(w+z) \leq v(w) v(z) \quad \forall w, z \in \mathbb{R}^{2 d} .
$$

(3) $v$ satisfies the Gelfand-Raikov-Shilov (GRS) condition:

$$
\lim _{n \rightarrow \infty} v(n z)^{\frac{1}{n}}=1 \quad \forall z \in \mathbb{R}^{2 d} .
$$

Every weight of the form $v(z)=e^{a|z|^{b}}(1+|z|)^{s} \log ^{r}(e+|z|)$, with real parameters $a, r, s \geq 0$ and $0 \leq b<1$, is admissible. Weights of particular interest are provided by

$$
v_{s}(z)=\langle z\rangle^{s}=\left(1+|z|^{2}\right)^{\frac{s}{2}}, \quad z \in \mathbb{R}^{2 d}, s \geq 0
$$


Observe that, for $s \geq 0$, the weight function $v_{s}$ is equivalent to the submultiplicative weight $(1+|\cdot|)^{s}$, that is, there exist $C_{1}, C_{2}>0$ such that

$$
C_{1} v_{s}(z) \leq(1+|z|)^{s} \leq C_{2} v_{s}(z), \quad z \in \mathbb{R}^{2 d} .
$$

A positive, even weight function $m$ on $\mathbb{R}^{2 d}$ is called $v$-moderate if $m\left(z_{1}+z_{2}\right) \leq$ $C v\left(z_{1}\right) m\left(z_{2}\right)$ for all $z_{1}, z_{2} \in \mathbb{R}^{2 d}$. We simply write $\mathcal{M}_{v}$ for the class of $v$-moderate weights.

Here and elsewhere the conjugate exponent $p^{\prime}$ of $p \in[1, \infty]$ is defined by $1 / p+$ $1 / p^{\prime}=1$. Moreover, in order to remain in the framework of tempered distributions, in what follows we shall consider weight functions $m$ on $\mathbb{R}^{d}$ or $\mathbb{R}^{2 d}$ satisfying the following condition

$$
m(z) \geq 1, \quad \forall z \in \mathbb{R}^{d} \quad \text { or } \quad m(z) \gtrsim\langle z\rangle^{-N} .
$$

for a suitable $N \in \mathbb{N}$. The same holds for weights on $\mathbb{R}^{2 d}$.

Modulation Spaces. Given a non-zero window $g \in \mathcal{S}\left(\mathbb{R}^{d}\right)$, a $v$-moderate weight function $m$ on $\mathbb{R}^{2 d}$ satisfying (15), and $1 \leq p, q \leq \infty$, the modulation space $M_{m}^{p, q}\left(\mathbb{R}^{d}\right)$ consists of all tempered distributions $f \in \mathcal{S}^{\prime}\left(\mathbb{R}^{d}\right)$ such that $V_{g} f \in L_{m}^{p, q}\left(\mathbb{R}^{2 d}\right)$ (weighted mixed-norm space). The norm on $M_{m}^{p, q}$ is

$$
\|f\|_{M_{m}^{p, q}}=\left\|V_{g} f\right\|_{L_{m}^{p, q}}=\left(\int_{\mathbb{R}^{d}}\left(\int_{\mathbb{R}^{d}}\left|V_{g} f(x, \omega)\right|^{p} m(x, \omega)^{p} d x\right)^{q / p} d \omega\right)^{1 / q} .
$$

If $p=q$, we write $M_{m}^{p}$ instead of $M_{m}^{p, p}$, and if $m(z) \equiv 1$ on $\mathbb{R}^{2 d}$, then we write $M^{p, q}$ and $M^{p}$ for $M_{m}^{p, q}$ and $M_{m}^{p, p}$.

Then $M_{m}^{p, q}\left(\mathbb{R}^{d}\right)$ is a Banach space whose definition is independent of the choice of the window $g$. The class of admissible windows can be extended to $M_{v}^{1}$, as stated below ([Grö06, Thm. 11.3.7]).

Theorem 2.1. Let $m$ be a v-moderate weight satisfying (15) and $g \in M_{v}^{1} \backslash\{0\}$, then $\left\|V_{g} f\right\|_{L_{m}^{p, q}}$ is an equivalent norm for $M_{m}^{p, q}\left(\mathbb{R}^{d}\right)$.

Hence, given any $g \in M_{v}^{1}\left(\mathbb{R}^{d}\right)$ and $f \in M_{m}^{p, q}$ we have the norm equivalence

$$
\|f\|_{M_{m}^{p, q}} \asymp\left\|V_{g} f\right\|_{L_{m}^{p, q}} .
$$

The previous equivalence will be heavily exploited in this work. We recall the inversion formula for the STFT (see [Grö01, Proposition 11.3.2]): assume $g \in$ $M_{v}^{1}\left(\mathbb{R}^{d}\right) \backslash\{0\}, f \in M_{m}^{p, q}\left(\mathbb{R}^{d}\right)$, with $m$ satisfying (15) then

$$
f=\frac{1}{\|g\|_{2}^{2}} \int_{\mathbb{R}^{2 d}} V_{g} f(z) \pi(z) g d z,
$$

and the equality holds in $M_{m}^{p, q}\left(\mathbb{R}^{d}\right)$. 
The adjoint operator of $V_{g}$, defined by

$$
V_{g}^{*} F(t)=\int_{\mathbb{R}^{2 d}} F(z) \pi(z) g d z
$$

maps the Banach space $L_{m}^{p, q}\left(\mathbb{R}^{2 d}\right)$ into $M_{m}^{p, q}\left(\mathbb{R}^{d}\right)$. In particular, if $F=V_{g} f$ the inversion formula (17) reads

$$
\operatorname{Id}_{M_{m}^{p, q}}=\frac{1}{\|g\|_{2}^{2}} V_{g}^{*} V_{g}
$$

Wiener Amalgam Spaces. Fix $g \in \mathcal{S}\left(\mathbb{R}^{d}\right) \backslash\{0\}$. Consider even weight functions $u, w$ on $\mathbb{R}^{d}$ satisfying (15). Then the Wiener amalgam space $W\left(\mathcal{F} L_{u}^{p}, L_{w}^{q}\right)\left(\mathbb{R}^{d}\right)$ is the space of distributions $f \in \mathcal{S}^{\prime}\left(\mathbb{R}^{d}\right)$ such that

$$
\|f\|_{W\left(\mathcal{F} L_{u}^{p}, L_{w}^{q}\right)\left(\mathbb{R}^{d}\right)}:=\left(\int_{\mathbb{R}^{d}}\left(\int_{\mathbb{R}^{d}}\left|V_{g} f(x, \omega)\right|^{p} u^{p}(\omega) d \omega\right)^{q / p} w^{q}(x) d x\right)^{1 / q}<\infty
$$

(obvious modifications for $p=\infty$ or $q=\infty$ ). Using the fundamental identity of time-frequency analysis (10), we can write $\left|V_{g} f(x, \omega)\right|=\left|V_{\hat{g}} \hat{f}(\omega,-x)\right|=\left|\mathcal{F}\left(\hat{f} T_{\omega} \overline{\hat{g}}\right)(-x)\right|$ and $($ recall $u(x)=u(-x))$

$$
\|f\|_{M_{u \otimes w}^{p, q}}=\left(\int_{\mathbb{R}^{d}}\left\|\hat{f} T_{\omega} \overline{\hat{g}}\right\|_{\mathcal{F} L_{u}^{p}}^{q} w^{q}(\omega) d \omega\right)^{1 / q}=\|\hat{f}\|_{W\left(\mathcal{F} L_{u}^{p}, L_{w}^{q}\right)} .
$$

Hence the Wiener amalgam spaces under our consideration are simply the image under Fourier transform of modulation spaces

$$
\mathcal{F}\left(M_{u \otimes w}^{p, q}\right)=W\left(\mathcal{F} L_{u}^{p}, L_{w}^{q}\right) .
$$

Using the relation (19) and Theorem 2.1 one can easily infer the following issue.

Theorem 2.2. Let $w_{i}$ be a $v_{i}$-moderate weight on $\mathbb{R}^{d}, i=1,2$ satisfying (15) and $g \in W\left(\mathcal{F} L_{w_{1}}^{1}, L_{w_{2}}^{1}\right)$. Then \|\|$V_{g} f(x, \cdot)\left\|_{L_{w_{2}}^{p}}\right\|_{L_{w_{1}}^{q}}$ is an equivalent norm for $f \in$ $W\left(\mathcal{F} L_{w_{1}}^{p}, L_{w_{2}}^{q}\right)$. In other words, the class of admissible windows for $f \in W\left(\mathcal{F} L_{w_{1}}^{p}, L_{w_{2}}^{q}\right)$ can be extended to $W\left(\mathcal{F} L_{w_{1}}^{1}, L_{w_{2}}^{1}\right)$.

2.2. $\tau$-Pseudodifferential Operators. In the spirit of the time-frequency-oriented presentation given in Grö01, let us introduce the $\tau$-pseudodifferential operators by means of superposition of time-frequency shifts, i.e.

$$
\mathrm{Op}_{\tau}(\sigma) f(x)=\int_{\mathbb{R}^{2 d}} \hat{\sigma}(\omega, u) e^{-2 \pi i(1-\tau) \omega u}\left(T_{-u} M_{\omega} f\right)(x) d u d \omega, \quad x \in \mathbb{R}^{d},
$$

for any $\tau \in[0,1]$. The symbol $\sigma$ and the function $f$ belong to suitable function spaces, to be determined in order for the previous expression to make sense. For instance, with obvious modifications to [Grö01, Lem. 14.3.1], we immediately get that $\mathrm{Op}_{\tau}(\sigma)$ maps $\mathcal{S}\left(\mathbb{R}^{d}\right)$ to $\mathcal{S}^{\prime}\left(\mathbb{R}^{2 d}\right)$ whenever $\sigma \in \mathcal{S}^{\prime}\left(\mathbb{R}^{2 d}\right)$. 
Notice that the representation (20) is a well-defined absolutely convergent integral if $\hat{\sigma} \in L^{1}\left(\mathbb{R}^{2 d}\right)$ and $f \in \mathcal{S}\left(\mathbb{R}^{d}\right)$. Under these assumptions, easy computations allow to retrieve the usual integral form of $\tau$-pseudodifferential operators, i.e.,

$$
\mathrm{Op}_{\tau}(\sigma) f(x)=\int_{\mathbb{R}^{2 d}} e^{2 \pi i(x-y) \omega} \sigma((1-\tau) x+\tau y, \omega) f(y) d y d \omega
$$

We finally aim to represent $\mathrm{Op}_{\tau}(\sigma)$ as an integral operator of the form

$$
\mathrm{Op}_{\tau}(\sigma) f(x)=\int_{\mathbb{R}^{2 d}} k(x, y) f(y) d y
$$

Let us introduce the operator $\mathfrak{T}_{\tau}$ acting on functions on $\mathbb{R}^{2 d}$ as

$\mathfrak{T}_{\tau} F(x, y)=F(x+\tau y, x-(1-\tau) y), \quad \mathfrak{T}_{\tau}^{-1} F(x, y)=F((1-\tau) x+\tau y, x-y)$, and denote by $\mathcal{F}_{i}, i=1,2$, the partial Fourier transform with respect to the $i$-th $d$-dimensional variable (it is then clear that $\mathcal{F}=\mathcal{F}_{1} \mathcal{F}_{2}$ ). Notice that

$$
k(x, y)=\mathfrak{T}_{\tau}^{-1} \mathcal{F}_{2}^{-1} \sigma(x, y)=\mathcal{F}_{1}^{-1} \hat{\sigma}((1-\tau) x+\tau y, y-x) .
$$

Since the operators $\mathfrak{T}_{\tau}$ and $\mathcal{F}_{i}$ are continuous bijections on $\mathcal{S}\left(\mathbb{R}^{2 d}\right)$, the kernel $k$ is well-defined (as a tempered distribution) also for symbols in $\mathcal{S}^{\prime}\left(\mathbb{R}^{2 d}\right)$ and we can finally recover the representation by duality given in the Introduction. We resume these remarks in the following result.

Proposition 2.4. For any symbol $\sigma \in \mathcal{S}^{\prime}\left(\mathbb{R}^{2 d}\right)$ and any real $\tau \in[0,1]$, the map $\mathrm{Op}_{\tau}(\sigma): \mathcal{S}\left(\mathbb{R}^{d}\right) \rightarrow \mathcal{S}\left(\mathbb{R}^{d}\right)$ is defined as integral operator with distributional kernel

$$
k=\mathfrak{T}_{\tau}^{-1} \mathcal{F}_{2}^{-1} \sigma \in \mathcal{S}^{\prime}\left(\mathbb{R}^{2 d}\right),
$$

meaning that, for any $f, g \in \mathcal{S}\left(\mathbb{R}^{d}\right)$,

$$
\left\langle\mathrm{Op}_{\tau}(\sigma) f, g\right\rangle=\langle k, g \otimes \bar{f}\rangle .
$$

In particular, since the representation

$$
W_{\tau}(f, g)(x, \omega)=\mathcal{F}_{2} \mathfrak{T}_{\tau}(f \otimes \bar{g})(x, \omega)
$$

holds for $f, g \in \mathcal{S}\left(\mathbb{R}^{d}\right)$, we have

$$
\left\langle\mathrm{Op}_{\tau}(\sigma) f, g\right\rangle=\left\langle\sigma, W_{\tau}(g, f)\right\rangle .
$$

The issues discussed insofar can be summed up in the following claim, whose proof is an application of the celebrated Schwartz's kernel theorem (see for instance [Grö01, Theorem 14.3.4]).

Theorem 2.3. Let $T: \mathcal{S}\left(\mathbb{R}^{d}\right) \rightarrow \mathcal{S}^{\prime}\left(\mathbb{R}^{d}\right)$ be a continuous linear operator. There exist tempered distributions $k, \sigma, F \in \mathcal{S}^{\prime}\left(\mathbb{R}^{d}\right)$ and $\tau \in[0,1]$ such that $T$ admits the following representations:

(i) as an integral operator: $\langle T f, g\rangle=\langle k, g \otimes \bar{f}\rangle$ for any $f, g \in \mathcal{S}\left(\mathbb{R}^{d}\right) ;$ 
(ii) as a $\tau$-pseudodifferential operator $T=\mathrm{Op}_{\tau}(\sigma)$ with symbol $\sigma$;

(iii) as a superposition (in a weak sense) of time-frequency shifts :

$$
T=\int_{\mathbb{R}^{2 d}} F(x, \omega) e^{2(1-\tau) \pi i x \omega} T_{x} M_{\omega} \mathrm{d} x \mathrm{~d} \omega .
$$

The relations among $k, \sigma$ and $F$ are the following:

$$
\sigma=\mathcal{F}_{2} \mathfrak{T}_{\tau} k, \quad F=\mathcal{I}_{2} \hat{\sigma},
$$

where $\mathcal{I}_{2}$ denotes the reflection in the second d-dimensional variable (i.e. $\mathcal{I}_{2} G(x, \omega)=$ $\left.G(x,-\omega),(x, \omega) \in \mathbb{R}^{2 d}\right)$.

\section{Covariance Property of $\tau$-Wigner Distribution FunCtions}

The proof of the following lemmas is a matter of computation.

Lemma 3.1. For $\tau \in(0,1)$, we define the operator

$$
A_{\tau}: f(t) \mapsto \mathcal{I} f\left(\frac{1-\tau}{\tau} t\right),
$$

where $\mathcal{I}$ is the reflection operator $(\mathcal{I} g(t)=g(-t))$. Then, for any $z=\left(z_{1}, z_{2}\right) \in \mathbb{R}^{2 d}$,

$$
\begin{aligned}
& \pi\left(z_{1}, z_{2}\right) A_{\tau}=A_{\tau} \pi\left(-\frac{1-\tau}{\tau} z_{1},-\frac{\tau}{1-\tau} z_{2}\right), \\
& A_{\tau} \pi\left(z_{1}, z_{2}\right)=\pi\left(-\frac{\tau}{1-\tau} z_{1},-\frac{1-\tau}{\tau} z_{2}\right) A_{\tau} .
\end{aligned}
$$

Lemma 3.2 ([BDDOC10, Lemma 6.2]). The $\tau$-Wigner distribution admits a representation as short-time Fourier transform for $\tau \in(0,1)$ :

$$
W_{\tau}(f, g)(x, \omega)=\frac{1}{\tau^{d}} e^{2 \pi i \frac{1}{\tau} x \omega} V_{A_{\tau} g} f\left(\frac{1}{1-\tau} x, \frac{1}{\tau} \omega\right)
$$

Let us define the linear map $\mathcal{B}_{\tau}: \mathbb{R}^{2 d} \rightarrow \mathbb{R}^{2 d}$ as

$$
\mathcal{B}_{\tau}(x)=\mathcal{B}_{\tau}\left(x_{1}, x_{2}\right)=\left(\frac{1}{1-\tau} x_{1}, \frac{1}{\tau} x_{2}\right)=\left(\begin{array}{cc}
\frac{1}{1-\tau} I_{d \times d} & 0_{d \times d} \\
0_{d \times d} & \frac{1}{\tau} I_{d \times d}
\end{array}\right)\left(\begin{array}{l}
x_{1} \\
x_{2}
\end{array}\right) .
$$

Under this convention, Eq. (24) becomes

$$
W_{\tau}(f, g)(x, \omega)=\frac{1}{\tau^{d}} e^{2 \pi i \frac{1}{\tau} x \omega} V_{A_{\tau} g} f\left(\mathcal{B}_{\tau}(x, \omega)\right) .
$$

As it is customary, we use the symbol $\mathcal{B}_{\tau}$ also to denote the matrix

$$
\mathcal{B}_{\tau}=\left(\begin{array}{cc}
\frac{1}{1-\tau} I_{d \times d} & 0_{d \times d} \\
0_{d \times d} & \frac{1}{\tau} I_{d \times d}
\end{array}\right)
$$


Proposition 3.3. For $\tau \in(0,1)$, $z=\left(z_{1}, z_{2}\right), w=\left(w_{1}, w_{2}\right) \in \mathbb{R}^{2 d}$, set

$$
\mathcal{T}_{\tau}(z, w)=\left(\begin{array}{c}
(1-\tau) z_{1}+\tau w_{1} \\
\tau z_{2}+(1-\tau) w_{2}
\end{array}\right) \quad z=\left(z_{1}, z_{2}\right), w=\left(w_{1}, w_{2}\right) \in \mathbb{R}^{2 d}
$$

Then, for $f, g \in \mathcal{S}\left(\mathbb{R}^{d}\right)$ we have

$$
W_{\tau}(\pi(z) f, \pi(w) g)(x, \omega)=c_{\tau} M_{J(z-w)} T_{\mathcal{T}_{\tau}(z, w)} W_{\tau}(f, g)(x, \omega)
$$

where the phase factor $c_{\tau}$ is given by

$$
c_{\tau}=e^{2 \pi i\left[\left(z_{1}-w_{1}\right)\left(\tau z_{2}+(1-\tau) w_{2}\right)\right]} .
$$

Equivalently, formula (29) reads

(31) $W_{\tau}(\pi(z) f, \pi(w) g)(u)=c_{\tau} \cdot \pi\left(\mathcal{T}_{\tau}(z, w), J(z-w)\right) W_{\tau}(f, g)(u), \quad u \in \mathbb{R}^{2 d}$,

where the phase factor $c_{\tau}$ is defined in (30).

Proof. Formula (24) gives

$$
W_{\tau}(\pi(z) f, \pi(w) g)(x, \omega)=\frac{1}{\tau^{d}} e^{2 \pi i \frac{1}{\tau} x \omega} V_{A_{\tau} \pi(w) g}(\pi(z) f)\left(\frac{1}{1-\tau} x, \frac{1}{\tau} \omega\right)
$$

For what concerns the short-time Fourier transform above, we can write, using (23) and then (8),

$$
\begin{aligned}
V_{A_{\tau} \pi(w) g}(\pi(z) f) & \left(\frac{1}{1-\tau} x, \frac{1}{\tau} \omega\right)=\left\langle\pi(z) f, \pi\left(\frac{1}{1-\tau} x, \frac{1}{\tau} \omega\right) A_{\tau} \pi(w) g\right\rangle \\
& =\left\langle\pi(z) f, \pi\left(\frac{1}{1-\tau} x, \frac{1}{\tau} \omega\right) \pi\left(-\frac{\tau}{1-\tau} w_{1},-\frac{1-\tau}{\tau} w_{2}\right) A_{\tau} g\right\rangle \\
& =\left\langle\pi(z) f, e^{-2 \pi i\left(\frac{1}{1-\tau} x\right)\left(-\frac{1-\tau}{\tau} w_{2}\right)} \pi\left(\frac{x-\tau w_{1}}{1-\tau}, \frac{\omega-(1-\tau) w_{2}}{\tau}\right) A_{\tau} g\right\rangle \\
& =e^{-2 \pi i \frac{1}{\tau} x w_{2}}\left\langle M_{z_{2}} T_{z_{1}} f, M_{\frac{\omega-(1-\tau) w_{2}}{\tau}} T_{\frac{x-\tau w_{1}}{1-\tau}} A_{\tau} g\right\rangle \\
& =e^{-2 \pi i \frac{1}{\tau} x w_{2}}\left\langle f, T_{-z_{1}} M_{-z_{2}} M_{\frac{\omega-(1-\tau) w_{2}}{\tau}} T_{\frac{x-\tau w_{1}}{1-\tau}} A_{\tau} g\right\rangle \\
& =e^{-2 \pi i \frac{1}{\tau} x w_{2}}\left\langle f, T_{-z_{1}} M_{\frac{\omega-(1-\tau) w_{2}-\tau w_{1}}{\tau}} T_{\frac{x-\tau w_{1}}{1-\tau}} A_{\tau} g\right\rangle .
\end{aligned}
$$


The commutation relations for TF-shifts (7) give

$$
\begin{aligned}
& V_{A_{\tau} \pi(w) g}(\pi(z) f)\left(\frac{1}{1-\tau} x, \frac{1}{\tau} \omega\right) \\
& =e^{-2 \pi i \frac{1}{\tau} x w_{2}}\left\langle f, e^{-2 \pi i\left(-z_{1}\right)\left(\frac{\omega-(1-\tau) w_{2}-\tau z_{2}}{\tau}\right)} M_{\frac{\omega-(1-\tau) w_{2}-\tau z_{2}}{\tau}} T_{\frac{x-\tau w_{2}-(1-\tau) z_{1}}{1-\tau}} A_{\tau} g\right\rangle \\
& =e^{-2 \pi i \frac{1}{\tau} x w_{2}} e^{-2 \pi i \frac{1}{\tau} z_{1}\left(\omega-(1-\tau) w_{2}-\tau z_{2}\right)}\left\langle f, M_{\frac{\omega-(1-\tau) w_{2}-\tau z_{2}}{\tau}} T_{\frac{x-\tau w_{1}-(1-\tau) z_{1}}{1-\tau}} A_{\tau} g\right\rangle \\
& =e^{-2 \pi i \frac{1}{\tau} z w_{2}} e^{-2 \pi i \frac{1}{\tau} z_{1}\left(\omega-(1-\tau) w_{2}-\tau z_{2}\right)} \\
& \times V_{A_{\tau} g} f\left(\frac{x-\left[(1-\tau) z_{1}+\tau w_{1}\right]}{1-\tau}, \frac{\omega-\left[\tau z_{2}+(1-\tau) w_{2}\right]}{\tau}\right) .
\end{aligned}
$$

Coming back to the original problem, we can write

$$
\begin{aligned}
W_{\tau}(\pi(z) f, \pi(w) g)(x, \omega)= & \frac{1}{\tau^{d}} e^{2 \pi i \frac{1}{\tau} x \omega} e^{-2 \pi i \frac{1}{\tau} x w_{2}} e^{-2 \pi i \frac{1}{\tau} z_{1}\left(\omega-(1-\tau) w_{2}-\tau z_{2}\right)} \\
& \times V_{A_{\tau} g} f\left(\frac{x-\left[(1-\tau) z_{1}+\tau w_{1}\right]}{1-\tau}, \frac{\omega-\left[\tau z_{1}+(1-\tau) w_{2}\right]}{\tau}\right) .
\end{aligned}
$$

Working on the phase factors, we obtain

$$
\begin{aligned}
& W_{\tau}(\pi(z) f, \pi(w) g)(x, \omega) \\
& =e^{-2 \pi i \frac{1}{\tau} x w_{2}} e^{-2 \pi i \frac{1}{\tau} z_{1}\left(\omega-(1-\tau) w_{2}-\tau z_{2}\right)} e^{2 \pi i \frac{1}{\tau} x\left(\tau z_{2}+(1-\tau) w_{2}\right)} e^{2 \pi i \frac{1}{\tau} \omega\left((1-\tau) z_{1}+\tau w_{1}\right)} \\
& \quad \times e^{-2 \pi i \frac{1}{\tau}\left[(1-\tau) z_{1}+\tau w_{1}\right]\left[\tau z_{2}+(1-\tau) w_{2}\right]} T_{\mathcal{T}_{\tau}(z, w)} W_{\tau}(f, g)(x, \omega) \\
& =c_{\tau} M_{J(z-w)} T_{\mathcal{T}_{\tau}(z, w)} W_{\tau}(f, g)(x, \omega),
\end{aligned}
$$

where $c_{\tau}$ is defined in (30). Finally, formula (31) is an easy computation.

Remark 3.1. (i) Notice that $\mathcal{T}_{\tau}(z, w)=\mathcal{T}_{1-\tau}(w, z)$ for every $z, w \in \mathbb{R}^{2 d}$. Moreover, $\mathcal{T}_{\tau}$ is well-defined for any $\tau \in[0,1]$.

(ii) The proof of the covariance property heavily relies on the representation (24) and on the properties of the operator $A_{\tau}$, which are both well-defined only when $\tau \in(0,1)$. We shall see in the sequel that the same formula (31) holds for the end-points $\tau=0$ and $\tau=1$.

We now state the covariance property for the Rihaczek distribution: as announced in the remark above, we can retrieve it by the naive substitution $\tau=0$ in (29) or, equivalently, (31).

Proposition 3.4. Consider $f, g \in \mathcal{S}\left(\mathbb{R}^{d}\right)$. For $z=\left(z_{1}, z_{2}\right), w=\left(w_{1}, w_{2}\right) \in \mathbb{R}^{2 d}$,

$$
W_{0}(\pi(z) f, \pi(w) g)(x, \omega)=c_{0} e^{2 \pi i\left[(x, \omega) J\left(z_{1}-w_{1}, z_{2}-w_{2}\right)\right]} W_{0}(f, g)\left(x-z_{1}, \omega-w_{2}\right),
$$

where $c_{0}=e^{2 \pi i\left(z_{1}-w_{1}\right) w_{2}}$ is a phase factor. 
Equivalently, formula (32) reads

$$
W_{0}(\pi(z) f, \pi(w) g)=c_{0} \cdot \pi\left(\left(z_{1}, w_{2}\right), J(z-w)\right) W_{0}(f, g) .
$$

Proof. We have

$$
\begin{aligned}
W_{0}\left(\pi\left(z_{1}, z_{2}\right) f, \pi\left(w_{1}, w_{2}\right) g\right)(x, \omega)= & e^{-2 \pi i x \omega} M_{z_{2}} T_{z_{1}} f \cdot \overline{\mathcal{F}\left(M_{w_{2}} T_{w_{1}} g\right)(\omega)} \\
= & e^{-2 \pi i x \omega} e^{2 \pi i x z_{2}} f\left(x-z_{1}\right) \overline{T_{w_{2}} M_{-w_{1}} \hat{g}(\omega)} \\
= & e^{-2 \pi i x \omega} e^{2 \pi i x z_{2}} e^{2 \pi i w_{1}\left(\omega-w_{2}\right)} f\left(x-z_{1}\right) \overline{\hat{g}\left(\omega-w_{2}\right)} \\
= & e^{2 \pi i w_{2}\left(z_{1}-w_{1}\right)} e^{2 \pi i x\left(z_{2}-w_{2}\right)} e^{-2 \pi i \omega\left(z_{1}-w_{1}\right)} \\
& \times W_{0}(f, g)\left(x-z_{1}, \omega-w_{2}\right),
\end{aligned}
$$

as desired.

We finally state the covariance property for the conjugate Rihaczek distribution, whose proof follows almost at once from the preceding result. It is again important to notice that it can be inferred by the naive substitution $\tau=1$ in (29).

Proposition 3.5. Consider $f, g \in \mathcal{S}\left(\mathbb{R}^{d}\right)$. For $z=\left(z_{1}, z_{2}\right), w=\left(w_{1}, w_{2}\right) \in \mathbb{R}^{2 d}$, we have

$$
W_{1}(\pi(z) f, \pi(w) g)(x, \omega)=c_{1} e^{2 \pi i\left[(x, \omega) J\left(z_{1}-w_{1}, z_{2}-w_{2}\right)\right]} W_{1}(f, g)\left(x-w_{1}, \omega-z_{2}\right),
$$

where $c_{1}=e^{2 \pi i\left(z_{1}-w_{1}\right) z_{2}}$ is a phase factor. Equivalently, formula (33) reads

$$
W_{1}(\pi(z) f, \pi(w) g)=c_{1} \cdot \pi\left(\left(w_{1}, z_{2}\right), J(z-w)\right) W_{1}(f, g) .
$$

Proof. It follows by the covariance property for the Rihaczek distribution:

$$
\begin{aligned}
W_{1}(\pi(z) f, \pi(w) g)(x, \omega) & =\overline{W_{0}(\pi(w) g, \pi(z) f)}(x, \omega) \\
& =e^{-2 \pi i\left(w_{1}-z_{1}\right) z_{2}} e^{2 \pi i\left[(x, \omega) J\left(z_{1}-w_{1}, z_{2}-w_{2}\right)\right]} \overline{W_{0}(g, f)}\left(x-w_{1}, \omega-z_{2}\right) \\
& =e^{2 \pi i\left(z_{1}-w_{1}\right) z_{2}} e^{2 \pi i\left[(x, \omega) J\left(z_{1}-w_{1}, z_{2}-w_{2}\right)\right]} W_{1}(f, g)\left(x-w_{1}, \omega-z_{2}\right) .
\end{aligned}
$$

This completes the proof.

\section{Almost diagonalization}

Lemma 3.1 in Grö06 has his analogous for $\tau$-pseudodifferential operators as follows.

Lemma 4.1. Fix a non-zero window $\varphi \in \mathcal{S}\left(\mathbb{R}^{d}\right)$ and set $\Phi_{\tau}=W_{\tau}(\varphi, \varphi)$ for $\tau \in$ $[0,1]$. Then, for $\sigma \in \mathcal{S}^{\prime}\left(\mathbb{R}^{2 d}\right)$,

$$
\left|\left\langle\mathrm{Op}_{\tau}(\sigma) \pi(z) \varphi, \pi(w) \varphi\right\rangle\right|=\left|V_{\Phi_{\tau}} \sigma\left(\mathcal{T}_{\tau}(z, w), J(w-z)\right)\right|=\left|V_{\Phi_{\tau}} \sigma(x, y)\right|
$$

and

$$
\left|V_{\Phi_{\tau}} \sigma(x, y)\right|=\left|\left\langle\mathrm{Op}_{\tau}(\sigma) \pi(z(x, y)) \varphi, \pi(w(x, y)) \varphi\right\rangle\right|
$$


for all $w, z, x, y \in \mathbb{R}^{2 d}$, where $\mathcal{T}_{\tau}$ is defined in (28) and

$$
z(x, y)=\left(\begin{array}{c}
x_{1}+(1-\tau) y_{2} \\
x_{2}-\tau y_{1}
\end{array}\right), \quad w(x, y)=\left(\begin{array}{c}
x_{1}-\tau y_{2} \\
x_{2}+(1-\tau) y_{1}
\end{array}\right)
$$

Proof. For $\tau \in(0,1)$, we use the time-frequency representation of $\tau$-Wigner pseudodifferential operators and the covariance property in Prop. 3.3.

$$
\begin{aligned}
\left\langle\mathrm{Op}_{\tau}(\sigma) \pi(z) \varphi, \pi(w) \varphi\right\rangle & =\left\langle\sigma, W_{\tau}(\pi(w) \varphi, \pi(z) \varphi)\right\rangle \\
& =\left\langle\sigma, c_{\tau} M_{J(w-z)} T_{\mathcal{T}_{\tau}(w, z)} W_{\tau}(\varphi, \varphi)\right\rangle \\
& =\overline{c_{\tau}} V_{\Phi_{\tau}} \sigma\left(\mathcal{T}_{\tau}(w, z), J(w-z)\right) .
\end{aligned}
$$

Formula (35) follows by setting $x=\mathcal{T}_{\tau}(w, z)$ and $y=J(w-z)$.

For what concerns the case $\tau=0$, we use formula (32) and calculate

$$
\begin{aligned}
\left\langle\mathrm{Op}_{0}(\sigma) \pi(z) \varphi, \pi(w) \varphi\right\rangle & =\left\langle\sigma, W_{0}(\pi(w) \varphi, \pi(z) \varphi)\right\rangle \\
& =\left\langle\sigma, c_{0} M_{J(w-z)} T_{\mathcal{T}_{0}(w, z)} W_{0}(\varphi, \varphi)\right\rangle \\
& =\overline{c_{0}} V_{\Phi_{0}} \sigma\left(\mathcal{T}_{0}(w, z), J(w-z)\right) .
\end{aligned}
$$

Formula (35) follows by setting $x=\mathcal{T}_{0}(w, z)$ and $y=J(w-z)$. Solving the system for $z$ and $w$ gives (36). The case $\tau=1$ follows the same pattern. Indeed, by (33),

$$
\begin{aligned}
\left\langle\mathrm{Op}_{1}(\sigma) \pi(z) \varphi, \pi(w) \varphi\right\rangle & =\left\langle\sigma, W_{1}(\pi(w) \varphi, \pi(z) \varphi)\right\rangle \\
& =\left\langle\sigma, c_{1} M_{J(w-z)} T_{\mathcal{T}_{1}(w, z)} W_{1}(\varphi, \varphi)\right\rangle \\
& =\overline{c_{1}} V_{\Phi_{1}} \sigma\left(\mathcal{T}_{1}(w, z), J(w-z)\right) .
\end{aligned}
$$

Lemma 4.2. Let $v$ be an admissible weight function on $\mathbb{R}^{2 d}$ and $1 \leq p \leq \infty$.

(i) If $\tau \in[0,1], f \in M_{v}^{1}\left(\mathbb{R}^{d}\right), g \in M_{v}^{p}\left(\mathbb{R}^{d}\right)$ we have $W_{\tau}(g, f) \in M_{1 \otimes v \circ J^{-1}}^{1, p}\left(\mathbb{R}^{2 d}\right)$, with

$$
\left\|W_{\tau}(g, f)\right\|_{M_{1 \otimes v 0 J^{-1}}^{1, p}} \lesssim_{\tau}\|f\|_{M_{v}^{1}}\|g\|_{M_{v}^{p}}
$$

(ii) If $\tau \in(0,1), f \in M_{v \circ \mathcal{U}_{\tau}}^{1}\left(\mathbb{R}^{d}\right), g \in M_{v}^{p}\left(\mathbb{R}^{d}\right)$, we have $W_{\tau}(g, f) \in W\left(\mathcal{F} L^{1}, L_{v \circ \mathcal{B}_{\tau}}^{p}\right)\left(\mathbb{R}^{2 d}\right)$, with

$$
\left\|W_{\tau}(g, f)\right\|_{W\left(\mathcal{F} L^{1}, L_{v \circ \mathcal{B}_{\tau}}^{p}\right)} \lesssim_{\tau}[\tau(1-\tau)]^{-d / p^{\prime}}\|f\|_{M_{v \circ \mathcal{U}_{\tau}}^{1}}\|g\|_{M_{v}^{p}}
$$

and the matrices $\mathcal{B}_{\tau}$ and $\mathcal{U}_{\tau}$ defined in (27) and (5), respectively. 
Proof. Fix $\varphi_{1}, \varphi_{2} \in \mathcal{S}\left(\mathbb{R}^{d}\right)$, thus $\Phi_{\tau}=W_{\tau}\left(\varphi_{1}, \varphi_{2}\right) \in \mathcal{S}\left(\mathbb{R}^{d}\right)$. Therefore, by Lemma 2.3 we can write (observe that the norm below may depend on $\tau$ )

$$
\begin{aligned}
A & :=\left\|W_{\tau}(g, f)\right\|_{M_{1 \otimes v 0 J^{-1}}^{1, p}\left(\mathbb{R}^{2 d}\right)}=\left(\int_{\mathbb{R}^{2 d}}\left(\int_{\mathbb{R}^{2 d}}\left|V_{\Phi_{\tau}} W_{\tau}(g, f)(z, \zeta)\right| d z\right)^{p} v\left(J^{-1} \zeta\right)^{p} d \zeta\right)^{\frac{1}{p}} \\
& =\left(\int_{\mathbb{R}^{2 d}}\left(\int_{\mathbb{R}^{2 d}}\left|V_{\varphi_{1}} g\left(z-\mathcal{A}_{1-\tau}^{\prime} \zeta\right)\right| \cdot\left|V_{\varphi_{2}} f\left(z+\mathcal{A}_{\tau}^{\prime} \zeta\right)\right| d z\right)^{p} v\left(J^{-1} \zeta\right)^{p} d \zeta\right)^{\frac{1}{p}} .
\end{aligned}
$$

The substitution $z^{\prime}=z+\mathcal{A}_{\tau}^{\prime} \zeta$, the properties of $\mathcal{A}_{\tau}^{\prime}$ provided in Lemma 2.2 (in particular $\mathcal{A}_{\tau}^{\prime}+\mathcal{A}_{1-\tau}^{\prime}=J$ ) and the fact that $v$ is an even function, give

$$
\begin{aligned}
A & =\left(\int_{\mathbb{R}^{2 d}}\left(\int_{\mathbb{R}^{2 d}}\left|V_{\varphi_{1}} g\left(z^{\prime}-J \zeta\right)\right| \cdot\left|V_{\varphi_{2}} f\left(z^{\prime}\right)\right| d z^{\prime}\right)^{p} v(-J \zeta)^{p} d \zeta\right)^{\frac{1}{p}} \\
& =\left(\int_{\mathbb{R}^{2 d}}\left[\left|V_{\varphi_{2}} f\right| *\left|V_{\varphi_{1}} g\right|^{*}(J \zeta)\right]^{p} v(J \zeta)^{p} d \zeta\right)^{\frac{1}{p}} \\
& =\left\|\left|V_{\varphi_{2}} f\right| *\left|V_{\varphi_{1}} g\right|^{*}\right\|_{L_{v}^{p}} \lesssim\left\|V_{\varphi_{2}} f\right\|_{L_{v}^{1}}\left\|V_{\varphi_{1}} g\right\|_{L_{v}^{p}} \asymp\|f\|_{M_{v}^{1}}\|g\|_{M_{v}^{p}} .
\end{aligned}
$$

Recall that $V_{\varphi_{1}} g^{*}(z)=\overline{V_{\varphi_{1}} g(-z)}$. In a similar fashion,

$$
\begin{aligned}
& B:=\left\|W_{\tau}(g, f)\right\|_{W\left(\mathcal{F} L^{1}, L_{v \circ \mathcal{B}_{\tau}}^{p}\right)\left(\mathbb{R}^{2 d}\right)}=\left(\int_{\mathbb{R}^{2 d}}\left(\int_{\mathbb{R}^{2 d}}\left|V_{\Phi_{\tau}}(g, f)(z, \zeta)\right| d \zeta\right)^{p} v\left(\mathcal{B}_{\tau} z\right)^{p} d z\right)^{\frac{1}{p}} \\
& =\left[\int_{\mathbb{R}^{2 d}}\left(\int_{\mathbb{R}^{2 d}}\left|V_{\varphi_{1}} g\left(z-\sqrt{\tau(1-\tau)} \mathcal{A}_{1-\tau} \zeta\right)\right| \cdot\left|V_{\varphi_{2}} f\left(z+\sqrt{\tau(1-\tau)} \mathcal{A}_{\tau} \zeta\right)\right| \mathrm{d} \zeta\right)^{p} v\left(\mathcal{B}_{\tau} z\right)^{p} \mathrm{~d} z\right]^{\frac{1}{p}} .
\end{aligned}
$$

Consider now the substitution $\eta=z-\sqrt{\tau(1-\tau)} \mathcal{A}_{1-\tau} \zeta$; by the properties of $\mathcal{A}_{\tau}$ provided in Lemma 2.2, we can write

$$
B=[\tau(1-\tau)]^{-d}\left(\int_{\mathbb{R}^{2 d}}\left(\int_{\mathbb{R}^{2 d}}\left|V_{\varphi_{1}} g(\eta)\right| \cdot\left|V_{\varphi_{2}} f\left(\mathcal{B}_{1-\tau} z+\mathcal{U}_{1-\tau} \eta\right)\right| d \eta\right)^{p} v\left(\mathcal{B}_{\tau} z\right)^{p} d z\right)^{\frac{1}{p}}
$$


Notice that $I-\mathcal{B}_{\tau}=\mathcal{U}_{\tau}$ and $\mathcal{U}_{\tau}^{-1}=\mathcal{U}_{1-\tau}($ cf. (15) $)$, hence

$$
\begin{aligned}
B & =[\tau(1-\tau)]^{-d}\left(\int_{\mathbb{R}^{2 d}}\left(\int_{\mathbb{R}^{2 d}}\left|V_{\varphi_{1}} g(\eta)\right| \cdot \mid V_{\varphi_{2}} f\left(\mathcal{U}_{1-\tau}\left(\eta+\mathcal{U}_{\tau} \mathcal{B}_{1-\tau} z\right) \mid d \eta\right)^{p} v\left(\mathcal{B}_{\tau} z\right)^{p} d z\right)^{\frac{1}{p}}\right. \\
& =[\tau(1-\tau)]^{-d}\left(\int_{\mathbb{R}^{2 d}}\left(\int_{\mathbb{R}^{2 d}}\left|V_{\varphi_{1}} g(\eta)\right| \cdot \mid V_{\varphi_{2}} f\left(\mathcal{U}_{1-\tau}\left(\eta-\mathcal{B}_{\tau} z\right) \mid d \eta\right)^{p} v\left(\mathcal{B}_{\tau} z\right)^{p} d z\right)^{\frac{1}{p}}\right. \\
& =[\tau(1-\tau)]^{-d}\left(\int_{\mathbb{R}^{2 d}}\left(\left|V_{\varphi_{1}} g\right| *\left|V_{\varphi_{2}} f\left(\mathcal{U}_{1-\tau} \cdot\right)\right|^{*}\right)^{p}\left(\mathcal{B}_{\tau} z\right) v\left(\mathcal{B}_{\tau} z\right)^{p} d z\right)^{\frac{1}{p}} \\
& =[\tau(1-\tau)]^{d\left(\frac{1}{p}-1\right)}\left\|\left|V_{\varphi_{1}} g\right| *\left|V_{\varphi_{2}} f\left(\mathcal{U}_{1-\tau} \cdot\right)\right|^{*}\right\|_{L_{v}^{p}} \\
& \lesssim[\tau(1-\tau)]^{d\left(\frac{1}{p}-1\right)}\left\|V_{\varphi_{2}} f\right\|_{L_{v o \mathcal{U}_{\tau}}^{1}}\left\|V_{\varphi_{1}} g\right\|_{L_{v}^{p}} \\
& \asymp[\tau(1-\tau)]^{d\left(\frac{1}{p}-1\right)}\|f\|_{M_{v o \mathcal{U}_{\tau}}^{1}}\|g\|_{M_{v}^{p}} .
\end{aligned}
$$

Thanks to this property we are able to extend to $\tau$-pseudodifferential operators, $\tau \in[0,1]$, the characterization (obtained only for Weyl operators) in Grö06, Theorem 3.2]. Namely,

Theorem 4.1. Let $v$ be an admissible weight function on $\mathbb{R}^{2 d}$. Consider $\varphi \in$ $M_{v}^{1}\left(\mathbb{R}^{d}\right) \backslash\{0\}$ and a lattice $\Lambda \subseteq \mathbb{R}^{2 d}$ such that $\mathcal{G}(\varphi, \Lambda)$ is a Gabor frame for $L^{2}\left(\mathbb{R}^{d}\right)$. For any $\tau \in[0,1]$, the following properties are equivalent:

(i) $\sigma \in M_{1 \otimes v \circ J^{-1}}^{\infty, 1}\left(\mathbb{R}^{2 d}\right)$.

(ii) $\sigma \in \mathcal{S}^{\prime}\left(\mathbb{R}^{2 d}\right)$ and there exists a function $H_{\tau} \in L_{v}^{1}\left(\mathbb{R}^{2 d}\right)$ such that

$$
\left|\left\langle\mathrm{Op}_{\tau}(\sigma) \pi(z) \varphi, \pi(w) \varphi\right\rangle\right| \leq H_{\tau}(w-z) \quad \forall w, z \in \mathbb{R}^{2 d} .
$$

(iii) $\sigma \in \mathcal{S}^{\prime}\left(\mathbb{R}^{2 d}\right)$ and there exists a sequence $h_{\tau} \in \ell_{v}^{1}(\Lambda)$ such that

$$
\left|\left\langle\mathrm{Op}_{\tau}(\sigma) \pi(\mu) \varphi, \pi(\lambda) \varphi\right\rangle\right| \leq h_{\tau}(\lambda-\mu) \quad \forall \lambda, \mu \in \Lambda .
$$

Proof. The proof follows the pattern of the corresponding one for Weyl operators Grö06, Theorem 3.2]. We detail here only the case $(i) \Rightarrow(i i)$, to show where the $\tau$-dependence of the controlling function $H_{\tau}$ comes from. The case of the sequence $h_{\tau}$ is similar. Consider $\varphi \in M_{v}^{1}\left(\mathbb{R}^{d}\right)$ as in the assumptions and set $\Phi_{\tau}=W_{\tau}(\varphi, \varphi) \in$ $M_{1 \otimes v \circ J^{-1}}^{1}\left(\mathbb{R}^{2 d}\right)$, by Lemma 4.2, part $(i)$. This implies that the short-time Fourier transform $V_{\Phi_{\tau}} \sigma$ is well-defined for $\sigma \in M_{1 \otimes v \circ J^{-1}}^{\infty, 1}\left(\mathbb{R}^{2 d}\right)$ (cf. Grö01, Theorem 11.3.7]). We now define $\tilde{H}_{\tau}(v)=\sup _{u \in \mathbb{R}^{2 d}}\left|V_{\Phi_{\tau}} \sigma(u, v)\right|$. By definition of $M_{1 \otimes v \circ J^{-1}}^{\infty, 1}\left(\mathbb{R}^{2 d}\right)$, we 
have $\tilde{H}_{\tau} \in L_{v \circ J^{-1}}^{1}\left(\mathbb{R}^{2 d}\right)$, so that Lemma 4.1 implies

$$
\begin{aligned}
\left|\left\langle\mathrm{Op}_{\tau}(\sigma) \pi(z) \varphi, \pi(w) \varphi\right\rangle\right| & =\left|V_{\Phi_{\tau}} \sigma\left(\mathcal{T}_{\tau}(z, w), J(w-z)\right)\right| \\
& \leq \sup _{u \in \mathbb{R}^{2 d}}\left|V_{\Phi_{\tau}} \sigma(u, J(w-z))\right| \\
& =\tilde{H}_{\tau}(J(w-z)) .
\end{aligned}
$$

Setting $H_{\tau}=\tilde{H}_{\tau} \circ J$ we obtain the claim.

Corollary 4.2. Under the hypotheses of Theorem 4.1, let $\tau \in[0,1]$ be fixed and assume that $T: \mathcal{S}\left(\mathbb{R}^{d}\right) \rightarrow \mathcal{S}^{\prime}\left(\mathbb{R}^{d}\right)$ is continuous and satisfies one of the following conditions:

(i) $|\langle T \pi(z) \varphi, \pi(w) \varphi\rangle| \leq H(w-z) \quad \forall w, z \in \mathbb{R}^{2 d}$ for some $H \in L_{v}^{1}$.

(ii) $|\langle T \pi(\mu) \varphi, \pi(\lambda) \varphi\rangle| \leq h(\lambda-\mu) \quad \forall \lambda, \mu \in \Lambda$ for some $h \in \ell_{v}^{1}$.

Therefore, $T=\mathrm{Op}_{\tau}(\sigma)$ for some symbol $\sigma \in M_{v \circ J^{-1}}^{\infty, 1}\left(\mathbb{R}^{2 d}\right)$.

Proof. Theorem 2.3 implies that $T=\mathrm{Op}_{\tau}(\sigma)$ for some $\tau \in[0,1]$ and some symbol $\sigma \in \mathcal{S}^{\prime}\left(\mathbb{R}^{2 d}\right)$. Then, the claim immediately follows from Theorem 4.1.

For $\tau \in(0,1)$ (but also for extremal values, see Theorem 4.7] in the sequel), we are able to prove a similar characterization for symbols in the Wiener amalgam space $W\left(\mathcal{F} L^{\infty}, L_{v}^{1}\right)$ via almost diagonalization.

Theorem 4.3. Let $v$ be an admissible weight function on $\mathbb{R}^{2 d}$. Consider $\varphi \in \mathcal{S}\left(\mathbb{R}^{d}\right) \backslash$ $\{0\}$. For any $\tau \in(0,1)$, the following properties are equivalent:

(i) $\sigma \in W\left(\mathcal{F} L^{\infty}, L_{v \circ \mathcal{B}_{\tau}}^{1}\right)\left(\mathbb{R}^{2 d}\right)$.

(ii) $\sigma \in \mathcal{S}^{\prime}\left(\mathbb{R}^{2 d}\right)$ and there exists a function $H_{\tau} \in L_{v}^{1}\left(\mathbb{R}^{2 d}\right)$ such that

$$
\left|\left\langle\mathrm{Op}_{\tau}(\sigma) \pi(z) \varphi, \pi(w) \varphi\right\rangle\right| \leq H_{\tau}\left(w-\mathcal{U}_{\tau} z\right) \quad \forall w, z \in \mathbb{R}^{2 d},
$$

where the matrices $\mathcal{B}_{\tau}$ and $\mathcal{U}_{\tau}$ are defined in (27) and (15), respectively.

Proof. $(i) \Rightarrow(i i)$. If $\varphi \in \mathcal{S}\left(\mathbb{R}^{d}\right) \subset M_{v}^{1}\left(\mathbb{R}^{d}\right) \cap M_{v o \mathcal{U}_{\tau}\left(\mathbb{R}^{d}\right)}^{1}, \varphi \neq 0$, then by Lemma 4.2, part $(i i)$, with $\Phi_{\tau}=W_{\tau}(\varphi, \varphi) \in W\left(\mathcal{F} L^{1}, L_{v \circ \mathcal{B}_{\tau}}^{1}\right)$. Take $\sigma \in W\left(\mathcal{F} L^{\infty}, L_{v \circ \mathcal{B}_{\tau}}^{1}\right)$, then $V_{\Phi_{\tau}} \sigma$ is well defined (cf. Theorem 2.2) and

$$
\tilde{H}_{\tau}(x)=\sup _{y \in \mathbb{R}^{2 d}}\left|V_{\Phi_{\tau}} \sigma(x, y)\right| \in L_{v \circ \mathcal{B}_{\tau}}^{1}\left(\mathbb{R}^{2 d}\right) .
$$

From Lemma 4.1 we infer

$$
\begin{aligned}
\left|\left\langle\mathrm{Op}_{\tau}(\sigma) \pi(z) \varphi, \pi(w) \varphi\right\rangle\right| & =\left|V_{\Phi_{\tau}} \sigma\left(\mathcal{T}_{\tau}(w, z), J(w-z)\right)\right| \\
& \leq \sup _{y \in \mathbb{R}^{2 d}}\left|V_{\Phi_{\tau}} \sigma\left(\mathcal{T}_{\tau}(w, z), y\right)\right| \\
& =\tilde{H}_{\tau}\left(\mathcal{T}_{\tau}(w, z)\right)
\end{aligned}
$$


Notice that

$$
\mathcal{B}_{\tau}\left(\mathcal{T}_{\tau}(w, z)\right)=\left(\begin{array}{c}
w_{1}+\frac{\tau}{1-\tau} z_{1} \\
w_{2}+\frac{1-\tau}{\tau} z_{2}
\end{array}\right)=w-\mathcal{U}_{\tau} z
$$

and thus $\tilde{H}_{\tau}\left(\mathcal{T}_{\tau}(w, z)\right)=\tilde{H}_{t}\left(\mathcal{B}_{\tau}^{-1}\left(w-\mathcal{U}_{\tau} z\right)\right)$. Define $H_{\tau}=\tilde{H}_{\tau} \circ \mathcal{B}_{\tau}^{-1}$, then $H_{\tau} \in$ $L_{v}^{1}\left(\mathbb{R}^{2 d}\right)$ since $\left\|H_{\tau}\right\|_{L_{v}^{1}}=\left\|\tilde{H}_{\tau} \circ \mathcal{B}_{\tau}^{-1}\right\|_{L_{v}^{1}} \asymp\left\|\tilde{H}_{\tau}\right\|_{L_{v \circ \mathcal{B}_{\tau}}^{1}}<\infty$.

Let us now show $(i i) \Rightarrow(i)$. Assume that $\sigma \in \mathcal{S}^{\prime}\left(\mathbb{R}^{2 d}\right)$ and that $\mathrm{Op}_{\tau}(\sigma)$ is almost diagonalized by the time-frequency shifts with dominating function $H_{\tau} \in L_{v}^{1}\left(\mathbb{R}^{2 d}\right)$ as in (41). Equation (35) and the results in the previous step (in particular, set $\tilde{H}_{\tau}=H_{\tau} \circ \mathcal{B}_{\tau}$ and then $H_{\tau}\left(w-\mathcal{U}_{\tau} z\right)=\tilde{H}_{\tau}\left(\mathcal{T}_{\tau}(w, z)\right)$ with $\mathcal{U}_{\tau}$ in (15)) allow to write

$$
\begin{aligned}
\left|V_{\Phi_{\tau}} \sigma(x, y)\right| & =\left|\left\langle\mathrm{Op}_{\tau}(\sigma) \pi(z(x, y)) \varphi, \pi(w(x, y)) \varphi\right\rangle\right| \\
& \leq H_{\tau}\left(w(x, y)-\mathcal{U}_{\tau} z(x, y)\right) \\
& =\tilde{H}_{\tau}\left(\mathcal{T}_{\tau}(w(x, y), z(x, y))\right)
\end{aligned}
$$

and since by construction $\mathcal{T}_{\tau}(w(x, y), z(x, y))=x$, we finally have

$$
\left|V_{\Phi_{\tau}} \sigma(x, y)\right| \leq \tilde{H}_{\tau}(x), \quad \forall x \in \mathbb{R}^{2 d} .
$$

Therefore,

$$
\begin{aligned}
\|\sigma\|_{W\left(\mathcal{F} L^{\infty}, L_{v \circ \mathcal{B} \tau}^{1}\right)} & =\int_{\mathbb{R}^{2 d}} \sup _{y \in \mathbb{R}^{2 d}}\left|V_{\Phi_{\tau}} \sigma(x, y)\right| v\left(\mathcal{B}_{\tau}(x)\right) d x \\
& \leq \int_{\mathbb{R}^{2 d}} \tilde{H}_{\tau}(x) v\left(\mathcal{B}_{\tau}(x)\right) d x \leq\left\|\tilde{H}_{\tau}\right\|_{L_{v \circ \mathcal{B}}^{1}} \asymp\left\|H_{\tau}\right\|_{L_{v}^{1}}<\infty
\end{aligned}
$$

and thus $\sigma \in W\left(\mathcal{F} L^{\infty}, L_{v \circ \mathcal{B}_{\tau}}^{1}\right)$.

Example 4.4. Consider $\sigma=\delta \in W\left(\mathcal{F} L^{\infty}, L^{1}\right)\left(\mathbb{R}^{2 d}\right)$. In this case, using formula (29),

$$
\begin{aligned}
\left|\left\langle\mathrm{Op}_{\tau}(\delta) \pi(z) \varphi, \pi(w) \varphi\right\rangle\right| & =\left|\left\langle\delta, W_{\tau}(\pi(w) \varphi, \pi(z) \varphi)\right\rangle\right| \\
& =\left|\left\langle\delta, c_{\tau} M_{J(w-z)} T_{\mathcal{T}_{\tau}(w, z)} W_{\tau}(\varphi, \varphi)\right\rangle\right| \\
& =\left|T_{\mathcal{T}_{\tau}(w, z)} W_{\tau}(\varphi, \varphi)(0)\right| \\
& =\mid W_{\tau}(\varphi, \varphi)\left(-\mathcal{T}_{\tau}(w, z)\right) \\
& =\left|W_{\tau}(\varphi, \varphi)\left(-\mathcal{B}_{\tau}^{-1}\left(w-\mathcal{U}_{\tau} z\right)\right)\right|
\end{aligned}
$$

Choosing $H_{\tau}(z)=\left|W_{\tau}(\varphi, \varphi)\left(-\mathcal{B}_{\tau}^{-1}(z)\right)\right|$ we obtain (41), which reduces to an equality in this case.

We remark that in this framework the discrete characterization of Theorem 4.1 is lost. The main obstruction is the following: for a given lattice $\Lambda$, the inclusion $\mathcal{U}_{\tau} \Lambda \subseteq \Lambda$ holds if and only if $\mathcal{U}_{\tau}=\mathcal{U}_{1 / 2}=-I_{2 d \times 2 d}$, the (minus) identity matrix. In this particular framework, the matrix $\mathcal{B}_{1 / 2}$ becomes $\mathcal{B}_{1 / 2}=2 I_{2 d \times 2 d}$ and Theorem 4.3 can be improved as follows. 
Corollary 4.5. Let $v$ be an admissible weight function on $\mathbb{R}^{2 d}$ and set $v_{2}=v \circ 2 I$. Consider $\varphi \in \mathcal{S}\left(\mathbb{R}^{d}\right) \backslash\{0\}$ such that $\mathcal{G}(\varphi, \Lambda)$ is a Gabor frame for $L^{2}\left(\mathbb{R}^{d}\right)$. For Weyl operators, the following properties are equivalent:

(i) $\sigma \in W\left(\mathcal{F} L^{\infty}, L_{v_{2}}^{1}\right)\left(\mathbb{R}^{2 d}\right)$.

(ii) $\sigma \in \mathcal{S}^{\prime}\left(\mathbb{R}^{2 d}\right)$ and there exists a function $H \in L_{v}^{1}\left(\mathbb{R}^{2 d}\right)$ such that

$$
\left|\left\langle\mathrm{Op}_{1 / 2}(\sigma) \pi(z) \varphi, \pi(w) \varphi\right\rangle\right| \leq H(w+z) \quad \forall w, z \in \mathbb{R}^{2 d},
$$

(iii) $\sigma \in \mathcal{S}^{\prime}\left(\mathbb{R}^{2 d}\right)$ and there exists a sequence $h \in \ell_{v}^{1}(\Lambda)$ such that

$$
\left|\left\langle\mathrm{Op}_{1 / 2}(\sigma) \pi(\mu) \varphi, \pi(\lambda) \varphi\right\rangle\right| \leq h(\lambda+\mu) \quad \forall \lambda, \mu \in \Lambda .
$$

Proof. The equivalence $(i) \Leftrightarrow(i i)$ is the consequence of Theorem 4.3, whereas the implications $(i) \Rightarrow($ iii $)$ and $(i i i) \Rightarrow(i)$ follow the same pattern as in the proof of Grö06].

Therefore, the symmetry of the Weyl quantization appears more powerful in this framework.

Remark 4.6. Observe that the function space for the window $\varphi$ in Theorem 4.3 and Corollary 4.5 can be extended from $\mathcal{S}\left(\mathbb{R}^{d}\right) \backslash\{0\}$ to $M_{v}^{1}\left(\mathbb{R}^{d}\right) \cap M_{v \circ \mathcal{U}_{\tau}}^{1}\left(\mathbb{R}^{d}\right)$, cf. Lemma 4.2.

The statement and the proof of Theorem 4.3 are not well suited for the degenerate cases $\tau=0$ and $\tau=1$ (notice that $\mathcal{B}_{\tau}$ and $\mathcal{U}_{\tau}$ are not even defined), thus the boundedness results which follow cannot be reproduced in this context. However, a weaker version of that result can be proved for any $\tau \in[0,1]$.

Theorem 4.7. Consider an admissible weight $v$ on $\mathbb{R}^{2 d}$ and a non-zero window $\varphi \in \mathcal{S}\left(\mathbb{R}^{d}\right)$. For any $\tau \in[0,1]$, the following properties are equivalent:

(i) $\sigma \in W\left(\mathcal{F} L^{\infty}, L_{v}^{1}\right)\left(\mathbb{R}^{2 d}\right)$.

(ii) $\sigma \in \mathcal{S}^{\prime}\left(\mathbb{R}^{2 d}\right)$ and there exists a function $H_{\tau} \in L_{v}^{1}\left(\mathbb{R}^{2 d}\right)$ such that

$$
\left|\left\langle\mathrm{Op}_{\tau}(\sigma) \pi(z) \varphi, \pi(w) \varphi\right\rangle\right| \leq H_{\tau}\left(\mathcal{T}_{\tau}(w, z)\right),
$$

where $\mathcal{T}_{\tau}$ is defined in (28).

Proof. $(i) \Rightarrow($ ii $)$. Take $\sigma \in W\left(\mathcal{F} L^{\infty}, L_{v}^{1}\right)$ and set

$$
H_{\tau}(x)=\sup _{y \in \mathbb{R}^{2 d}}\left|V_{\Phi_{\tau}} \sigma(x, y)\right| \text {. }
$$

By definition of $W\left(\mathcal{F} L^{\infty}, L_{v}^{1}\right)$ we have $H_{\tau} \in L_{v}^{1}\left(\mathbb{R}^{2 d}\right)$. Since the linear map $(w, z) \mapsto$ $J(w-z)$ is surjective, from Lemma 4.1 we infer 


$$
\begin{aligned}
\left|\left\langle\mathrm{Op}_{\tau}(\sigma) \pi(z) \varphi, \pi(w) \varphi\right\rangle\right| & =\left|V_{\Phi_{\tau}} \sigma\left(\mathcal{T}_{\tau}(w, z), J(w-z)\right)\right| \\
& \leq \sup _{y \in \mathbb{R}^{2 d}}\left|V_{\Phi_{\tau}} \sigma\left(\mathcal{T}_{\tau}(w, z), y\right)\right| \\
& =H_{\tau}\left(\mathcal{T}_{\tau}(w, z)\right) .
\end{aligned}
$$

This gives the claim.

$(i i) \Rightarrow(i)$. Assume that $\sigma \in \mathcal{S}^{\prime}\left(\mathbb{R}^{2 d}\right)$ and that $\mathrm{Op}_{\tau}(\sigma)$ is almost diagonalized by the time-frequency shifts with dominating function $H_{\tau} \in L_{v}^{1}\left(\mathbb{R}^{2 d}\right)$ as in (41). Equation (35) and the results in the previous step allow to write

$$
\begin{aligned}
\left|V_{\Phi_{\tau}} \sigma(x, y)\right| & =\left|\left\langle\mathrm{Op}_{\tau}(\sigma) \pi(z(x, y)) \varphi, \pi(w(x, y)) \varphi\right\rangle\right| \\
& \leq H_{\tau}\left(\mathcal{T}_{\tau}(w(x, y), z(x, y))\right),
\end{aligned}
$$

and since by construction $\mathcal{T}_{\tau}(w(x, y), z(x, y))=x$, we finally have

$$
\left|V_{\Phi_{\tau}} \sigma(x, y)\right| \leq H_{\tau}(x) \quad \forall x \in \mathbb{R}^{2 d}
$$

Therefore,

$$
\begin{aligned}
\|\sigma\|_{W\left(\mathcal{F} L^{\infty}, L_{v}^{1}\right)} & =\int_{\mathbb{R}^{2 d}} \sup _{y \in \mathbb{R}^{2 d}}\left|V_{\Phi_{\tau}} \sigma(x, y)\right| v(x) d x \\
& \leq \int_{\mathbb{R}^{2 d}} H_{\tau}(x) v(x) d x \leq\left\|H_{\tau}\right\|_{L_{v}^{1}}<\infty
\end{aligned}
$$

that is $\sigma \in W\left(\mathcal{F} L^{\infty}, L_{v}^{1}\right)$.

\section{Boundedness RESUlts}

5.1. Boundedness on Modulation Spaces. As a consequence of the diagonalization provided by Theorem 4.3, we infer the boundedness of $\tau$-pseudodifferential operators with symbols in the Wiener amalgam space $W\left(\mathcal{F} L^{\infty}, L_{v \circ \mathcal{B}_{\tau}}^{1}\right)$ on every modulation space, as follows.

Theorem 5.1. Fix $m \in \mathcal{M}_{v}$ satisfying (15). For $\tau \in(0,1)$ consider a symbol $\sigma \in W\left(\mathcal{F} L^{\infty}, L_{v \circ \mathcal{B}_{\tau}}^{1}\right)\left(\mathbb{R}^{2 d}\right)$, with the matrix $\mathcal{B}_{\tau}$ defined in (27). Then the operator $\mathrm{Op}_{\tau}(\sigma)$ is bounded from $M_{m}^{p, q}\left(\mathbb{R}^{d}\right)$ to $M_{m o \mathcal{U}_{\tau}}^{p, q}\left(\mathbb{R}^{d}\right), 1 \leq p, q \leq \infty$.

Proof. The proof uses the techniques developed in [CGNR14, Theorem 3.3]. We fix $g(t)=e^{-\pi t^{2}} \in M_{v}^{1}\left(\mathbb{R}^{d}\right)$ for every admissible weight $v$. Observe that $\|g\|_{2}=1$, so that the inversion formula (18) is simply $V_{g}^{*} V_{g}=\mathrm{Id}$. Writing $T$ as

$$
T=V_{g}^{*} V_{g} T V_{g}^{*} V_{g}
$$

then $V_{g} T V_{g}^{*}$ is an integral operator with kernel

$$
K_{T}(w, z)=\langle T \pi(z) g, \pi(w) g\rangle, \quad w, z \in \mathbb{R}^{2 d} .
$$


By definition, $V_{g}$ is bounded from $M_{m}^{p}\left(\mathbb{R}^{d}\right)$ to $L_{m}^{p}\left(\mathbb{R}^{2 d}\right)$ and $V_{g}^{*}$ is bounded from $L_{m}^{p}\left(\mathbb{R}^{2 d}\right)$ to $M_{m}^{p}\left(\mathbb{R}^{d}\right)$. Hence, if $V_{g} T V_{g}^{*}$ is bounded from $L_{m}^{p}\left(\mathbb{R}^{2 d}\right)$ to $L_{m \circ \mathcal{U}_{\tau}}^{p}\left(\mathbb{R}^{2 d}\right)$, then $T$ is bounded from $M_{m}^{p}\left(\mathbb{R}^{d}\right)$ to $M_{m o \mathcal{U}_{\tau}}^{p}\left(\mathbb{R}^{d}\right)$. Observe that

$$
\mathcal{U}_{\tau} \circ \mathcal{B}_{1-\tau}=-\mathcal{B}_{\tau}
$$

so that $v \circ \mathcal{U}_{\tau} \circ \mathcal{B}_{1-\tau}=v \circ \mathcal{B}_{\tau}$, and recall that $\mathcal{U}_{1-\tau}^{-1}=\mathcal{U}_{\tau}$. Applying Theorem 4.3 with $1-\tau$ in place of $\tau$ and with the admissible weight $v \circ \mathcal{U}_{\tau}$ in place of $v$, for $F \in L_{m}^{p}\left(\mathbb{R}^{2 d}\right)$,

$$
\begin{aligned}
\left|V_{g} T V_{g}^{*} F(w)\right| & =\left|\int_{\mathbb{R}^{2 d}} K_{T}(w, z) F(z) d z\right| \leq \int_{\mathbb{R}^{2 d}}|F(z)| H_{1-\tau}\left(w-\mathcal{U}_{1-\tau} z\right) d z \\
& =\int_{\mathbb{R}^{2 d}}|F(z)|\left(H_{1-\tau} \circ \mathcal{U}_{1-\tau}\right)\left(\mathcal{U}_{\tau} w-z\right) d z \\
& =F *\left(H_{1-\tau} \circ \mathcal{U}_{1-\tau}\right)\left(\mathcal{U}_{\tau} w\right) .
\end{aligned}
$$

By Theorem 4.3, $H_{1-\tau}$ is in $L_{v \circ \mathcal{U}_{\tau}}^{1}\left(\mathbb{R}^{2 d}\right)$ and thus $H_{1-\tau} \circ \mathcal{U}_{1-\tau}$ is in $L_{v}^{1}\left(\mathbb{R}^{2 d}\right)$. Therefore, by Young's inequality $F *\left(H_{1-\tau} \circ \mathcal{U}_{1-\tau}\right) \in L_{m}^{p}\left(\mathbb{R}^{2 d}\right) * L_{v}^{1}\left(\mathbb{R}^{2 d}\right) \subset L_{m}^{p}\left(\mathbb{R}^{2 d}\right)$. This shows that $V_{g} T V_{g}^{*} F \in L_{m o \mathcal{U}_{\tau}}^{p}\left(\mathbb{R}^{2 d}\right)$, as desired.

If we limit the study to the polynomial weights $v_{s}$ defined in (14), then the theory of Fourier integral operators (FIOs) developed in [CGNR14 tells us further issues of Theorem 4.3.

We recall [CGNR14, Definition 1.1].

Definition 5.2. Given $\mathcal{A} \in \mathrm{Sp}(2 d, \mathbb{R}), g \in \mathcal{S}\left(\mathbb{R}^{d}\right)$, and $s \geq 0$, we say that a linear operator $T: \mathcal{S}\left(\mathbb{R}^{d}\right) \rightarrow \mathcal{S}^{\prime}\left(\mathbb{R}^{d}\right)$ is in the class $F I O\left(\mathcal{A}, v_{s}\right)$ if there exists a function $H \in L_{v_{s}}^{1}\left(\mathbb{R}^{2 d}\right)$ such that the kernel of $T$ with respect to time-frequency shifts satisfies the decay condition

$$
|\langle T \pi(z) g, \pi(w) g\rangle| \leq H(w-\mathcal{A} z), \quad \forall w, z \in \mathbb{R}^{2 d} .
$$

Moreover, the generalized metaplectic operators that can be represented as FIOs of type I are as follows (cf. [CGNR14, Theorem 5.1]):

Theorem 5.3. Let $s \geq 0$ and $\mathcal{A}=\left(\begin{array}{cc}A & B \\ C & D\end{array}\right) \in \operatorname{Sp}(2 d, \mathbb{R})$ with $\operatorname{det} A \neq 0$, and let $T: \mathcal{S}\left(\mathbb{R}^{d}\right) \rightarrow \mathcal{S}^{\prime}\left(\mathbb{R}^{d}\right)$ be a linear continuous operator. Then $T \in F I O\left(\mathcal{A}, v_{s}\right)$ if and only if $T$ is a FIO of type I, i.e.

$$
T f(x)=\int_{\mathbb{R}^{d}} e^{2 \pi i \Phi(x, \omega)} \sigma(x, \omega) \hat{f}(\omega) d \omega
$$

with the quadratic phase $\Phi(x, \omega)=\frac{1}{2} x C A^{-1} x+\omega A^{-1} x-\frac{1}{2} \eta A^{-1} B \omega$ and a symbol $\sigma \in M_{1 \otimes v_{s}}^{\infty, 1}\left(\mathbb{R}^{2 d}\right)$. 
Observe that $v_{s} \circ \mathcal{B}_{\tau} \asymp v_{s}$ (with the bounds depending on $\tau$ ). If the symbol $\sigma$ is in $W\left(\mathcal{F} L^{\infty}, L_{v_{s}}^{1}\right)$ then Theorem 4.3 says that (41) holds for a suitable function $H_{\tau} \in L_{v_{s}}^{1}$, so that the $\tau$-operator $\operatorname{Op}_{\tau}(\sigma)$ is in the class $F I O\left(\mathcal{U}_{\tau}, v_{s}\right)$. Moreover, the assumptions of Theorem 5.3 are satisfied and we can thus represent $\operatorname{Op}_{\tau}(\sigma)$ as a type I FIO with phase $\Phi(x, \omega)=-\frac{1-\tau}{\tau} \omega x$ as follows:

$$
\mathrm{Op}_{\tau}(\sigma) f(x)=\int_{\mathbb{R}^{d}} e^{-2 \pi i \frac{1-\tau}{\tau} \omega x} \rho(x, \omega) \hat{f}(\omega) d \omega,
$$

for a suitable symbol $\rho \in M_{1 \otimes v_{s}}^{\infty, 1}\left(\mathbb{R}^{d}\right)$. Finally, by Theorem 1.2 in [CGNR14] we obtain:

Corollary 5.4. If $\sigma \in W\left(\mathcal{F} L^{\infty}, L_{v_{s}}^{1}\right), s \geq 0$, then the operator $\operatorname{Op}_{\tau}(\sigma)$ is bounded on every modulation space $M_{v_{s}}^{p}\left(\mathbb{R}^{d}\right)$, for $1 \leq p \leq \infty$ and $\tau \in(0,1)$.

5.2. Boundedness on Wiener Amalgam Spaces. We now turn to consider $\tau$ pseudodifferential operators and their boundedness on Wiener amalgam spaces. We need two preliminary results.

Lemma 5.1. For any $\sigma \in \mathcal{S}^{\prime}\left(\mathbb{R}^{2 d}\right)$ and $\tau \in[0,1]$,

$$
\mathcal{F} \mathrm{Op}_{\tau}(\sigma) \mathcal{F}^{-1}=\mathrm{Op}_{1-\tau}\left(\sigma \circ J^{-1}\right) \text {. }
$$

Proof. It is a particular case of the symplectic covariance property of Shubin calculus, see [dG13, Proposition 10]. We also refer to the books [dG16, Hör85, Won98.

The proof of the following lemma is a straightforward computation.

Lemma 5.2. For any non-zero window $G \in \mathcal{S}\left(\mathbb{R}^{2 d}\right)$ and $\sigma \in \mathcal{S}^{\prime}\left(\mathbb{R}^{2 d}\right)$, then

$$
V_{G}(\sigma \circ J)(z, \zeta)=\left(V_{G \circ J-1} \sigma\right)(J z, J \zeta) .
$$

Therefore, for any $1 \leq p, q \leq \infty$, weights $u, v$ on $\mathbb{R}^{2 d}$ and $\tau \in[0,1]$ :

(i) $\sigma_{J}=\sigma \circ J \in M_{\left(u \circ J^{-1}\right) \otimes\left(v \circ J^{-1}\right)}^{p, q}\left(\mathbb{R}^{2 d}\right)$ if and only if $\sigma \in M_{u \otimes v}^{p, q}\left(\mathbb{R}^{2 d}\right)$. In particular,

$$
\sigma \in M_{1 \otimes v}^{\infty, 1}\left(\mathbb{R}^{2 d}\right) \Leftrightarrow \sigma_{J} \in M_{1 \otimes\left(v \circ J^{-1}\right)}^{\infty, 1}\left(\mathbb{R}^{2 d}\right) .
$$

(ii) $\sigma \in W\left(\mathcal{F} L_{u \circ J^{-1}}^{p}, L_{v \circ J^{-1}}^{q}\right)$ if and only if $\sigma_{J} \in W\left(\mathcal{F} L_{u}^{p}, L_{v}^{q}\right)$. In particular,

$$
\sigma \in W\left(\mathcal{F} L^{\infty}, L_{v \circ \mathcal{B}_{\tau} \circ J^{-1}}^{1}\right) \Leftrightarrow \sigma_{J} \in W\left(\mathcal{F} L^{\infty}, L_{v \circ \mathcal{B}_{\tau}}^{1}\right) .
$$

Proof. A direct computation shows that

$$
\begin{aligned}
V_{G} \sigma_{J}(z, \zeta) & =\left\langle\sigma_{J}, M_{\zeta} T_{z} G\right\rangle=\int e^{-2 \pi i \zeta(t, \omega)} \sigma_{J}(t, \omega) \overline{G\left(t-z_{1}, \omega-z_{2}\right)} d t d \omega \\
& =\int e^{-2 \pi i \zeta(t, \omega)} \sigma(J(t, \omega)) \overline{G\left(t-z_{1}, \omega-z_{2}\right)} d t d \omega .
\end{aligned}
$$


With the substitution $\left(t^{\prime}, \omega^{\prime}\right)=J(t, \omega)$, we have

$$
\begin{aligned}
V_{G} \sigma_{J}(z, \zeta) & =\int e^{-2 \pi i \zeta \cdot J^{-1}\left(t^{\prime}, \omega^{\prime}\right)} \sigma\left(t^{\prime}, \omega^{\prime}\right) \overline{G\left(J^{-1}\left[\left(t^{\prime}, \omega^{\prime}\right)-J z\right]\right)} d t^{\prime} d \omega^{\prime} \\
& =\left\langle\sigma, M_{J \zeta} T_{J z}\left(G \circ J^{-1}\right)\right\rangle=\left(V_{G \circ J^{-1}} \sigma\right)(J z, J \zeta) .
\end{aligned}
$$

Therefore,

$$
\begin{aligned}
\left\|\sigma_{J}\right\|_{M_{\left(u \circ J^{-1}\right) \otimes\left(v \circ J^{-1}\right)}^{p, q}} & =\left(\int_{\mathbb{R}^{2 d}}\left(\int_{\mathbb{R}^{2 d}}\left|V_{G} \sigma_{J}(z, \zeta)\right|^{p} u\left(J^{-1} z\right)^{p} d z\right)^{\frac{q}{p}} v\left(J^{-1} \zeta\right)^{q} d \zeta\right)^{\frac{1}{q}} \\
& =\left(\int_{\mathbb{R}^{2 d}}\left(\int_{\mathbb{R}^{2 d}}\left|V_{G \circ J^{-1}} \sigma(J z, J \zeta)\right|^{p} u\left(J^{-1} z\right)^{p} d z\right)^{\frac{q}{p}} v\left(J^{-1} \zeta\right)^{q} d \zeta\right)^{\frac{1}{q}} \\
& =\left(\int_{\mathbb{R}^{2 d}}\left(\int_{\mathbb{R}^{2 d}}\left|V_{G \circ J^{-1}} \sigma(z, \zeta)\right|^{p} u(-z)^{p} d z\right)^{\frac{q}{p}} v(-\zeta)^{q} d \zeta\right)^{\frac{1}{q}} \\
& \asymp\|\sigma\|_{M_{u \otimes v}^{p, q}} .
\end{aligned}
$$

where we used the even property of the weight functions. In a similar fashion,

$$
\begin{aligned}
\left\|\sigma_{J}\right\|_{W\left(\mathcal{F} L_{u}^{p}, L_{v}^{q}\right)} & =\left(\int_{\mathbb{R}^{2 d}}\left(\int_{\mathbb{R}^{2 d}}\left|V_{G} \sigma_{J}(z, \zeta)\right|^{p} u(\zeta)^{p} d \zeta\right)^{\frac{q}{p}} v(z)^{q} d z\right)^{\frac{1}{q}} \\
& =\left(\int_{\mathbb{R}^{2 d}}\left(\int_{\mathbb{R}^{2 d}}\left|V_{G \circ J^{-1}} \sigma(z, \zeta)\right|^{p} u\left(J^{-1} z\right)^{p} d z\right)^{\frac{q}{p}} v\left(J^{-1} \zeta\right)^{q} d \zeta\right)^{\frac{1}{q}} \\
& \asymp\|\sigma\|_{W\left(\mathcal{F} L_{u \circ J^{-1}}^{p}, L_{v \circ J^{-1}}^{q}\right)} .
\end{aligned}
$$

Another ingredient is the boundedness of $\tau$-pseudodifferential operators on modulation spaces, cf. Theorem 4.3 and Remark 4.5 in [Tof04] (see also Grö06, Theorem 4.1] for Weyl operators).

Theorem 5.5. Consider $m \in \mathcal{M}_{v}\left(\mathbb{R}^{2 d}\right)$ satisfying (15). For any $\tau \in[0,1]$ and $\sigma \in$ $M_{1 \otimes v \circ J^{-1}}^{\infty, 1}$ the operator $\mathrm{Op}_{\tau}(\sigma)$ is bounded on $M_{m}^{p, q}\left(\mathbb{R}^{d}\right)$, and there exists a constant $C_{\tau}>0$ such that

$$
\left\|\mathrm{Op}_{\tau}(\sigma)\right\|_{M_{m}^{p, q}} \leq C_{\tau}\|\sigma\|_{M_{1 \otimes v \circ J-1}^{\infty, 1}}
$$

We can now state the boundedness result for $\tau$-pseudodifferential operators on Wiener amalgam spaces. 
Theorem 5.6. Consider $m=m_{1} \otimes m_{2} \in \mathcal{M}_{v}\left(\mathbb{R}^{2 d}\right)$ satisfying (15). For any $\tau \in$ $[0,1]$ and $\sigma \in M_{1 \otimes v}^{\infty, 1}\left(\mathbb{R}^{2 d}\right)$, the operator $\operatorname{Op}_{\tau}(\sigma)$ is bounded on $W\left(\mathcal{F} L_{m_{1}}^{p}, L_{m_{2}}^{q}\right)\left(\mathbb{R}^{d}\right)$ with

$$
\left\|\mathrm{Op}_{\tau}(\sigma)\right\|_{W\left(\mathcal{F} L_{m_{1}, L_{m_{2}}^{q}}^{q}\right)} \leq C_{\tau}\|\sigma\|_{M_{1 \otimes v}^{\infty, 1}}
$$

for a suitable $C_{\tau}>0$.

Proof. Consider the following commutative diagram:

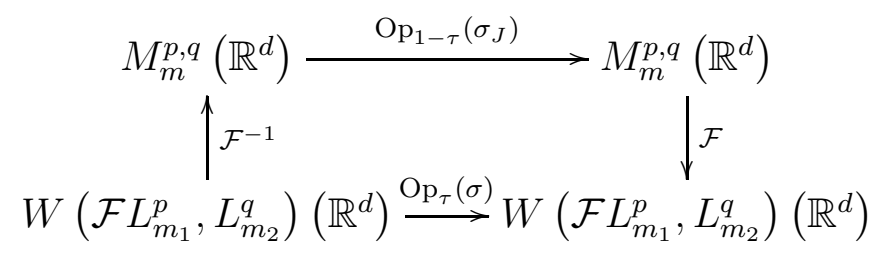

Indeed, since $\sigma \in M_{1 \otimes v}^{\infty, 1}\left(\mathbb{R}^{2 d}\right), \sigma_{J} \in M_{1 \otimes\left(v \circ J^{-1}\right)}^{\infty, 1}\left(\mathbb{R}^{2 d}\right)$ by Lemma 5.2. The operator $\mathrm{Op}_{1-\tau}\left(\sigma_{J}\right)$ is bounded on $M_{m}^{p, q}\left(\mathbb{R}^{d}\right)$ by virtue of Theorem 5.5 with $\tau^{\prime}=1-\tau \in[0,1]$ and the thesis follows at once thanks to the Lemma 5.1.

The same argument (with obvious modifications) allow to extend the boundedness result for $\tau$-pseudodifferential operators contained in Theorem 5.1 to Wiener amalgam spaces for symbols in suitable Wiener amalgam spaces.

Theorem 5.7. Consider $m=m_{1} \otimes m_{2} \in \mathcal{M}_{v}\left(\mathbb{R}^{2 d}\right)$ satisfying (15). For any $\tau \in(0,1)$ and $\sigma \in W\left(\mathcal{F} L^{\infty}, L_{v \circ \mathcal{B}_{\tau} \circ J^{-1}}^{1}\right)\left(\mathbb{R}^{2 d}\right)$, the operator $\mathrm{Op}_{\tau} \sigma$ is bounded from $W\left(\mathcal{F} L_{m_{1}}^{p}, L_{m_{2}}^{q}\right)\left(\mathbb{R}^{d}\right)$ to $W\left(\mathcal{F} L_{m_{1} \circ\left(\mathcal{U}_{\tau}\right)_{1}}^{p}, L_{m_{2} \circ\left(\mathcal{U}_{\tau}\right)_{2}}^{q}\right)\left(\mathbb{R}^{d}\right), 1 \leq p, q \leq \infty$, where

$$
\left(\mathcal{U}_{\tau}\right)_{1}(x)=-\frac{\tau}{1-\tau} x, \quad\left(\mathcal{U}_{\tau}\right)_{2}(x)=-\frac{1-\tau}{\tau} x, \quad x \in \mathbb{R}^{d} .
$$

Proof. Consider the following commutative diagram:

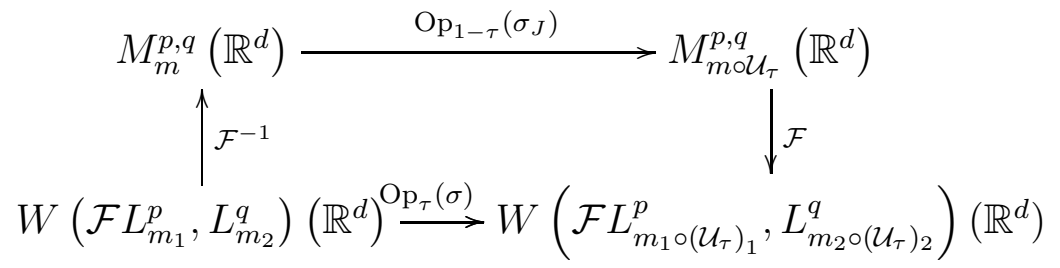

Indeed, since $\sigma \in W\left(\mathcal{F} L^{\infty}, L_{v \circ \mathcal{B}_{\tau} \circ J^{-1}}^{1}\right)\left(\mathbb{R}^{2 d}\right), \sigma_{J} \in W\left(\mathcal{F} L^{\infty}, L_{v \circ \mathcal{B}_{\tau}}^{1}\right)\left(\mathbb{R}^{2 d}\right)$ by Lemma 5.2. The operator $\mathrm{Op}_{1-\tau} \sigma_{J}$ is bounded by virtue of Theorem 5.1 with $\tau^{\prime}=1-\tau \in$ $(0,1)$ and the thesis follows at once thanks to the Lemma 5.1. 
The cases $\tau=0$ and $\tau=1$. Theorem 4.7 allows us to obtain some boundedness results for $\tau$-pseudodifferential operators with $\tau=0$ or $\tau=1$, having symbols in Wiener amalgam spaces.

Proposition 5.8. Assume $\sigma \in W\left(\mathcal{F} L^{\infty}, L^{1}\right)\left(\mathbb{R}^{d}\right)$. Then the Kohn-Nirenberg operator $\mathrm{Op}_{\mathrm{KN}}(\sigma)(\tau=0)$ is bounded on $M^{1, \infty}\left(\mathbb{R}^{d}\right)$.

Proof. Consider $H_{0}\left(\mathcal{T}_{0}(w, z)\right)$ with $H_{0} \in L^{1}\left(\mathbb{R}^{2 d}\right)$ as in Theorem 4.7. The integral operator $T_{H_{0}}$ with kernel $H_{0}\left(\mathcal{T}_{0}(w, z)\right)=H_{0}\left(w_{1}, z_{2}\right)$ can be written as

$$
T_{H_{0}} F(w)=\int_{\mathbb{R}^{2 d}} H_{0} \circ \mathcal{T}_{0}(w, z) F(z) d z=\int_{\mathbb{R}^{d}} \int_{\mathbb{R}^{d}} H\left(w_{1}, z_{2}\right) F\left(z_{1}, z_{2}\right) d z_{1} d z_{2} .
$$

It is immediate to notice that $T_{H_{0}}: L^{1, \infty}\left(\mathbb{R}^{2 d}\right) \rightarrow L^{1, \infty}\left(\mathbb{R}^{2 d}\right)$ is a bounded operator. Then, for a fixed non-zero window $g \in \mathcal{S}\left(\mathbb{R}^{d}\right)$, we have that

$$
T=V_{g}^{*} T_{H_{0}} V_{g}: M^{1, \infty}\left(\mathbb{R}^{d}\right) \rightarrow M^{1, \infty}\left(\mathbb{R}^{d}\right)
$$

is a bounded operator. The claim then follows.

Proposition 5.9. Assume $\sigma \in W\left(\mathcal{F} L^{\infty}, L^{1}\right)\left(\mathbb{R}^{d}\right)$. Then the operator "with right symbol" $\mathrm{Op}_{1}(\sigma)(\tau=1)$ is bounded on $W\left(\mathcal{F} L^{1}, L^{\infty}\right)\left(\mathbb{R}^{d}\right)$.

Proof. Again, we apply Theorem 4.7 and consider $H_{1}\left(\mathcal{T}_{1}(w, z)\right)$ with $H_{1} \in L^{1}\left(\mathbb{R}^{2 d}\right)$. The integral operator $T_{H}$ with kernel $H_{1}\left(\mathcal{T}_{1}(w, z)\right)=H_{1}\left(z_{1}, w_{2}\right)$ can be written as

$$
T_{H_{1}} F(w)=\int_{\mathbb{R}^{2 d}} H_{1} \circ \mathcal{T}_{1}(w, z) F(z) d z=\int_{\mathbb{R}^{d}} \int_{\mathbb{R}^{d}} H_{1}\left(z_{1}, w_{2}\right) F\left(z_{1}, z_{2}\right) d z_{1} d z_{2} .
$$

It is immediate to notice that $T_{H_{1}}: L_{z_{1}}^{\infty}\left(L_{z_{2}}^{1}\right)\left(\mathbb{R}^{2 d}\right) \rightarrow L_{w_{1}}^{\infty}\left(L_{w_{2}}^{1}\right)\left(\mathbb{R}^{2 d}\right)$ is a bounded operator. Then, for a fixed non-zero window $g \in \mathcal{S}\left(\mathbb{R}^{d}\right)$, we have that

$$
T=V_{g}^{*} T_{H_{1}} V_{g}: W\left(\mathcal{F} L^{1}, L^{\infty}\right)\left(\mathbb{R}^{d}\right) \rightarrow W\left(\mathcal{F} L^{1}, L^{\infty}\right)\left(\mathbb{R}^{d}\right)
$$

is a bounded operator. This concludes the proof.

The consequences of the almost diagonalization of $\tau$-operators are manifold. We notice that the results of this section can be extended by interpolation to symbols in $W\left(\mathcal{F} L^{p}, L^{q}\right)\left(\mathbb{R}^{2 d}\right)$, following the pattern of [CN16]. This subject with be further investigated in a subsequent paper.

\section{Algebra and Wiener properties}

The connection with the theory Fourier integral operators established in the previous section allows to investigate further properties of $\tau$-operators. First of all, notice that for any $\tau_{1}, \tau_{2} \in(0,1)$,

$$
\mathcal{U}_{\tau_{1}} \mathcal{U}_{\tau_{2}}=\left(\begin{array}{cc}
\frac{\tau_{1} \tau_{2}}{\left(1-\tau_{1}\right)\left(1-\tau_{2}\right)} I_{d \times d} & 0_{d \times d} \\
0_{d \times d} & \frac{\left(1-\tau_{1}\right)\left(1-\tau_{2}\right)}{\tau_{1} \tau_{2}} I_{d \times d}
\end{array}\right)
$$


In particular,

$$
\mathcal{U}_{\tau} \mathcal{U}_{1-\tau}=\mathcal{U}_{1-\tau} \mathcal{U}_{\tau}=I_{2 d \times 2 d}
$$

Therefore, composition properties of operators in the class $F I O\left(\mathcal{A}, v_{s}\right)$ (see CGNR14, Theorems 3.4] and Theorem 4.1) yield the following result.

Theorem 6.1 (Algebra property). For any $a, b \in W\left(\mathcal{F} L^{\infty}, L_{v_{s}}^{1}\right)$ and $\tau \in(0,1)$, there exists a symbol $c \in M_{1 \otimes v_{s}}^{\infty, 1}$ such that

$$
\mathrm{Op}_{\tau}(a) \mathrm{Op}_{1-\tau}(b)=\mathrm{Op}_{1 / 2}(c) \text {. }
$$

Remark 6.2. On the other hand, for any choice of $\tau_{1}, \tau_{2} \in(0,1)$, there is no $\tau \in$ $(0,1)$ such that $\mathcal{U}_{\tau_{1}} \mathcal{U}_{\tau_{2}}=\mathcal{U}_{\tau}$. This immediately implies that there is no $\tau$-quantization rule such that composition of $\tau$-operators with symbols in $W\left(\mathcal{F} L^{\infty}, L_{v_{s}}^{1}\right)$ has symbol in the same class. In a similar fashion, given $a \in W\left(\mathcal{F} L^{\infty}, L_{v_{s}}^{1}\right), b \in M_{1 \otimes v_{s}}^{\infty, 1}$ and $\tau, \tau_{0} \in(0,1)$, we have

$$
\mathrm{Op}_{\tau_{0}}(b) \mathrm{Op}_{\tau}(a)=\mathrm{Op}_{\tau}\left(c_{1}\right), \quad \mathrm{Op}_{\tau}(a) \mathrm{Op}_{\tau_{0}}(b)=\mathrm{Op}_{\tau}\left(c_{2}\right)
$$

for some $c_{1}, c_{2} \in W\left(\mathcal{F} L^{\infty}, L_{v_{s}}^{1}\right)$. This means that, for fixed quantization rules $\tau, \tau_{0}$, the amalgam space $W\left(\mathcal{F} L^{\infty}, L_{v_{s}}^{1}\right)$ is a bimodule over the algebra $M_{1 \otimes v_{s}}^{\infty, 1}$ under the laws

$$
\begin{aligned}
& M_{1 \otimes v_{s}}^{\infty, 1} \times W\left(\mathcal{F} L^{\infty}, L_{v_{s}}^{1}\right) \rightarrow W\left(\mathcal{F} L^{\infty}, L_{v_{s}}^{1}\right):(b, a) \mapsto c_{1}, \\
& W\left(\mathcal{F} L^{\infty}, L_{v_{s}}^{1}\right) \times M_{1 \otimes v_{s}}^{\infty, 1} \rightarrow W\left(\mathcal{F} L^{\infty}, L_{v_{s}}^{1}\right):(a, b) \mapsto c_{2},
\end{aligned}
$$

with $c_{1}$ and $c_{2}$ as before.

In conclusion, we provide a result whose proof easily follows by CGNR14, Theorem 3.7] and by noticing that $\mathcal{U}_{\tau}^{-1}=\mathcal{U}_{1-\tau}$ for any $\tau \in(0,1)$.

Theorem 6.3 (Wiener property). For any $\tau \in(0,1)$ and $a \in W\left(\mathcal{F} L^{\infty}, L_{v_{s}}^{1}\right)$ such that $\mathrm{Op}_{\tau}(a)$ is invertible on $L^{2}\left(\mathbb{R}^{d}\right)$, we have

$$
\mathrm{Op}_{\tau}(a)^{-1}=\mathrm{Op}_{1-\tau}(b)
$$

for some $b \in W\left(\mathcal{F} L^{\infty}, L_{v_{s}}^{1}\right)$.

\section{ACKnowledgments}

The first and second authors were partially supported by the Gruppo Nazionale per l'Analisi Matematica, la Probabilità e le loro Applicazioni (GNAMPA) of the Istituto Nazionale di Alta Matematica (INdAM). The authors wish to thank the referees for their suggestions, which improved the readability of the manuscript. 


\section{REFERENCES}

[BCDDO10] Paolo Boggiatto, Bui Kien Cuong, Giuseppe De Donno, and Alessandro Oliaro, Weighted integrals of Wigner representations, J. Pseudo-Differ. Oper. Appl. 1 (2010), no. 4, 401-415. MR 2747903

[BDDO10] Paolo Boggiatto, Giuseppe De Donno, and Alessandro Oliaro, Time-frequency representations of Wigner type and pseudo-differential operators, Trans. Amer. Math. Soc. 362 (2010), no. 9, 4955-4981. MR 2645057

[BDDOC10] Paolo Boggiatto, Giuseppe De Donno, Alessandro Oliaro, and Bui Kien Cuong, Generalized spectrograms and $\tau$-Wigner transforms, Cubo 12 (2010), no. 3, 171185. MR 2779380

[Bou95] A. Boulkhemair, $L^{2}$ estimates for pseudodifferential operators, Ann. Scuola Norm. Sup. Pisa Cl. Sci. (4) 22 (1995), no. 1, 155-183. MR 1315354

[Bou97a] _ Estimations $L^{2}$ précisées pour des intégrales oscillantes, Comm. Partial Differential Equations 22 (1997), no. 1-2, 165-184. MR 1434142

[Bou97b] _ Remarks on a Wiener type pseudodifferential algebra and Fourier integral operators, Math. Res. Lett. 4 (1997), no. 1, 53-67. MR 1432810

[CDNT18] Elena Cordero, Lorenza D'Elia, Fabio Nicola, and S. Ivan Trapasso, Boundedness Properties of $\tau$-Pseudodifferential Operators with Symbols in Wiener Amalgam Spaces on Modulation Spaces, Preprint (2018).

[CGNR13] Elena Cordero, Karlheinz Gröchenig, Fabio Nicola, and Luigi Rodino, Wiener algebras of fourier integral operators, Journal de mathématiques pures et appliquées 99 (2013), no. 2, 219-233.

[CGNR14] _ Generalized metaplectic operators and the Schrödinger equation with a potential in the Sjöstrand class, J. Math. Phys. 55 (2014), no. 8, 081506, 17. MR 3390695

[CN08] Elena Cordero and Fabio Nicola, Some new Strichartz estimates for the Schrödinger equation, J. Differential Equations 245 (2008), no. 7, 1945-1974. MR 2433493

[CN16] Sharp integral bounds for wigner distribution, International Mathematics Research Notices 2016 (2016), no. 00, 1-29.

[CNR09] Elena Cordero, Fabio Nicola, and Luigi Rodino, A few remarks on time-frequency analysis of gevrey, analytic and ultra-analytic functions, Pseudo-differential operators and symmetries: background analysis and advanced topics, vol. 2, Springer Science \& Business Media, 2009.

[DdGP14] Nuno Costa Dias, Maurice A. de Gosson, and João Nuno Prata, Maximal covariance group of Wigner transforms and pseudo-differential operators, Proc. Amer. Math. Soc. 142 (2014), no. 9, 3183-3192. MR 3223374

[dG11] Maurice A. de Gosson, Symplectic methods in harmonic analysis and in mathematical physics, Pseudo-Differential Operators. Theory and Applications, vol. 7, Birkhäuser/Springer Basel AG, Basel, 2011. MR 2827662

[dG13] _ Symplectic covariance properties for Shubin and Born-Jordan pseudodifferential operators, Trans. Amer. Math. Soc. 365 (2013), no. 6, 3287-3307. MR 3034466

[dG16] _ Born-Jordan quantization, Fundamental Theories of Physics, vol. 182, Springer, [Cham], 2016, Theory and applications. MR 3467497

[dG17] The Wigner transform, Advanced Textbooks in Mathematics, World Scientific Publishing Co. Pte. Ltd., Hackensack, NJ, 2017. MR 3643624 
[dGGR16] Maurice A. de Gosson, Karlheinz Gröchenig, and José Luis Romero, Stability of Gabor frames under small time Hamiltonian evolutions, Lett. Math. Phys. 106 (2016), no. 6, 799-809. MR 3500423

[Fei81] Hans G. Feichtinger, On a new Segal algebra, Monatsh. Math. 92 (1981), no. 4, 269-289. MR 643206

[Fei83] _ Modulation spaces on locally compact abelian groups, Universität Wien. Mathematisches Institut, 1983.

[Fei06] _ Modulation spaces: looking back and ahead, Sampl. Theory Signal Image Process. 5 (2006), no. 2, 109-140. MR 2233968

[GL08] Kanghui Guo and Demetrio Labate, Representation of Fourier integral operators using shearlets, J. Fourier Anal. Appl. 14 (2008), no. 3, 327-371. MR 2399105

[GR08] Karlheinz Gröchenig and Ziemowit Rzeszotnik, Banach algebras of pseudodifferential operators and their almost diagonalization, Annales de l'institut Fourier, vol. 58, 2008, pp. 2279-2314.

[Grö01] Karlheinz Gröchenig, Foundations of time-frequency analysis, Applied and Numerical Harmonic Analysis, Birkhäuser Boston, Inc., Boston, MA, 2001. MR 1843717

[Grö06] _ Time-frequency analysis of Sjöstrand's class, Rev. Mat. Iberoam. 22 (2006), no. 2, 703-724. MR 2294795

[Hör85] Lars Hörmander, The analysis of linear partial differential operators. iii, volume 274 of grundlehren der mathematischen wissenschaften [fundamental principles of mathematical sciences], Springer-Verlag, Berlin, 1985.

[Mey90] Y Meyer, Ondelettes et operateurs ii: Operateurs de calderon-zygmund.(wavelets and operators ii: Calderon-zygmund operators), Hermann, Editeurs des Sciences et des Arts, Paris (1990).

[MP13] S. Molahajloo and G. E. Pfander, Boundedness of pseudo-differential operators on $L^{p}$, Sobolev and modulation spaces, Math. Model. Nat. Phenom. 8 (2013), no. 1, 175-192. MR 3022988

[RT98] Richard Rochberg and Kazuya Tachizawa, Pseudodifferential operators, gabor frames, and local trigonometric bases, Gabor analysis and algorithms, Springer, 1998, pp. 171-192.

[RWZ16] Michael Ruzhansky, Baoxiang Wang, and Hua Zhang, Global well-posedness and scattering for the fourth order nonlinear Schrödinger equations with small data in modulation and Sobolev spaces, J. Math. Pures Appl. (9) 105 (2016), no. 1, 31-65. MR 3427938

[Sjö94] Johannes Sjöstrand, An algebra of pseudodifferential operators, Math. Res. Lett. 1 (1994), no. 2, 185-192. MR 95b:47065

[Sjö95] W Wiener type algebras of pseudodifferential operators, Séminaire sur les Équations aux Dérivées Partielles, 1994-1995, École Polytech., Palaiseau, 1995, pp. Exp. No. IV, 21. MR 1362552

[STW11] Mitsuru Sugimoto, Naohito Tomita, and Baoxiang Wang, Remarks on nonlinear operations on modulation spaces, Integral Transforms Spec. Funct. 22 (2011), no. 45, 351-358. MR 2801287

[Tof04] Joachim Toft, Continuity properties for modulation spaces, with applications to pseudo-differential calculus. II, Ann. Global Anal. Geom. 26 (2004), no. 1, 73-106. MR 2054576 
[WHHG11] Baoxiang Wang, Zhaohui Huo, Chengchun Hao, and Zihua Guo, Harmonic analysis method for nonlinear evolution equations. I, World Scientific Publishing Co. Pte. Ltd., Hackensack, NJ, 2011. MR 2848761

[Won98] M. W. Wong, Weyl transforms, Universitext, Springer-Verlag, New York, 1998. MR 1639461

Dipartimento di Matematica, Università di Torino, Dipartimento di Matematica, via Carlo Alberto 10, 10123 Torino, Italy

E-mail address: elena.cordero@unito.it

Dipartimento di Scienze Matematiche, Politecnico di Torino, corso Duca degli ABruzzi 24, 10129 TORINo, ItAly

E-mail address: fabio.nicola@polito.it

Dipartimento di Scienze Matematiche, Politecnico di Torino, corso Duca degli Abruzzi 24, 10129 Torino, Italy

E-mail address: salvatore.trapasso@polito.it 